The Astrophysical JouRnal, 479:200-221, 1997 April 10

(C) 1997. The American Astronomical Society. All rights reserved. Printed in U.S.A.

\title{
DYNAMICS OF BROAD EMISSION-LINE REGION IN NGC 5548: HYDROMAGNETIC WIND MODEL VERSUS OBSERVATIONS
}

\author{
MARK BotTorfF, Kirk T. Korista, AND IsAaC SHLOSMAN \\ Department of Physics and Astronomy, University of Kentucky, Lexington, KY 40506-0055; bottorff@pa.uky.edu, \\ korista@pa.uky.edu, shlosman@pa.uky.edu \\ AND \\ ROGER D. BLANDFORD \\ Theoretical Astrophysics 130-33, California Institute of Technology, Pasadena, CA 91125; rdb@tapir.caltech.edu \\ Received 1996 July 18; accepted 1996 October 17
}

\begin{abstract}
We analyze the results of long-term observations of the broad-line region (BLR) in the Seyfert 1 galaxy NGC 5548 and provide a critical comparison with the predictions of a hydromagnetically driven outflow model of Emmering, Blandford, \& Shlosman. We use this model to generate a time series of $\mathrm{C}$ IV line profiles that have responded to a time-varying continuum. Our modifications to the model include cloud emission anisotropy, cloud obscuration, a CLOUDY-generated emissivity function, and a narrow-line component which is added to the BLR component to generate the total line profiles. The model is driven with continuum input based on the monitoring campaigns of NGC 5548 reported in Clavel et al. and Korista et al., and the line strengths, profiles, and lags are compared with the observations.

Our model is able to reproduce the basic features of $\mathrm{C}$ IV line variability in this active galactic nucleus, i.e., time evolution of the profile shape and strength of the $\mathrm{C}$ IV emission line without varying the model parameters. The best-fit model provides the effective size, the dominant geometry, the emissivity distribution, and the three-dimensional velocity field of the C IV BLR and constrains the mass of the central black hole to $\sim 3 \times 10^{7} M_{\odot}$. The inner part of the wind in NGC 5548 appears to be responsible for the anisotropically emitted C IV line, while its outer part remains dusty and molecular, thus having similar spectral characteristics to a molecular torus, although its dynamics is fundamentally different.

In addition, our model predicts a differential response across the $\mathrm{C}$ IV line profile, producing a redside-first response in the relative velocity interval of $3000 \mathrm{~km} \mathrm{~s}^{-1}$ to $6000 \mathrm{~km} \mathrm{~s}^{-1}$ followed by the blue mid-wing and finally by the line core. Based on the comparison of data and model cross-correlation functions and one- and two-dimensional transfer functions, we find that the rotating outflow model is compatible with observations of the BLR in NGC 5548.

Subject headings: black hole physics — galaxies: active — galaxies: individual (NGC 5548) galaxies: nuclei — galaxies: Seyfert — line: formation
\end{abstract}

\section{INTRODUCTION}

Observations of active galactic nuclei (AGNs: quasars and Seyfert galaxies) have revealed broad emission lines superposed on a nonstellar continuum (Osterbrock 1996 and reference therein). Understanding broad emission-line regions (BLRs) of AGNs is clearly among the outstanding problems of modern research on galaxy evolution. Since their discovery, extensive effort has been invested in understanding the BLR geometry and its relationship to the central engine in AGNs and to the host galaxy. In the heart of the problem lies the origin of gas in the BLR and its physical state and dynamics. The emerging picture is that of photoionized clouds moving within $\sim 1 \mathrm{pc}$ of a central UV-to-X-ray continuum source. The observational support to this view consists of a rough proportionality between the emission lines and continuum, the inferred diverse ionization states of the emitting gas, and the similarity between the equivalent widths of hydrogen lines from the BLRs. Further constraints on the physical conditions within the emitting clouds and their kinematics come from the observed relative line ratios, line profiles, and their variability.

Although the resolution of the overall problem of BLRs has proved to be elusive due to a large phase space of possible solutions ill-constrained by observations, a con- sensus has emerged on a number of issues. First, the BLR clouds should be numerous as their line profiles appear to be smooth; the individual clouds are smaller than the central continuum source because deep intrinsic Lyman edges have never been observed. The clouds are optically thick at the Lyman limit, in Ly $\alpha$, and possibly other strong resonance lines, such as C IV $\lambda 1549$ (e.g., Netzer 1990). Second, systematic differences between the high and lowionization emission line (HILs and LILs) profiles, e.g., fullwidth at half-maxima (FWHM) and velocity shifts between line peaks (Gaskell 1982; Wilkes 1984; Collin-Souffrin \& Lasota 1988; Espey et al. 1989; Corbin 1990 and others), have suggested ionization stratification in the BLRs. Third, the results of recent monitoring campaigns of a number of AGNs have suggested much smaller sizes of line-emitting regions than previously anticipated and independently confirmed the ionization stratification of the BLRs (see review by Peterson 1993). Both the continuum and the emission lines in AGNs are observed to vary with time. The emission-line variability lags behind and represents the BLR response to the underlying continuum variations. The response or lag time differs from line to line. Blandford \& McKee (1982) proposed a "reverberation mapping" technique which uses the line and continuum variability data to infer the geometry and kinematics of the BLR. A number of 
phenomenological models utilizing simple geometries and motions of the ionized gas within the BLR have been calculated (e.g., Welsh \& Horne 1991; Perez, Robinson \& de la Fuente 1992a, 1992b; Robinson 1995). The long-term continuous monitoring and high-quality data produced in recent observations supply additional constraints on the theoretical models of the BLRs which can be tested now (Gondhalekar, Horne, \& Peterson 1994 and references therein).

All published models of BLRs fall within one or more of the following kinematic categories: infall, outflow, rotation, and random motion. It is understood theoretically that if the AGNs are accretion-powered, a strong inflow must be present, although it is by no means clear whether this has anything to do with the observed motion of the ionized gas in the BLRs. One possibility is that the inflowing gas stays molecular for as long as it is capable of shielding its dust from the external radiation field and internally generated heat. The dynamics of ionized gas, which is subject to a partial or full radiation field from the central continuum source, may be affected by the radiation force as well. Various acceleration mechanisms for the ionized gas in the BLRs have been proposed, such as continuum radiation pressure-driven clouds (Mathews 1986 and references therein), thermally driven winds (Weymann et al. 1982; Cassidy \& Raine 1996), line-driven winds from accretion disks (Shlosman, Vitello, \& Shaviv 1985; Murray et al. 1996), and magnetically driven winds (Emmering, Blandford, \& Shlosman 1992; Königl \& Kartje 1994). Alternatively, radial infall models have been tested in order to reproduce the observed emission-line profiles (Krolik \& London 1983), sometimes accompanied with random cloud motions (Kwan \& Carrol 1982). Rotating accretion disk models of BLRs have been explored as well (e.g., Shields 1978; Mathews 1982). A recent revival of the line-driven winds from accretion disks as being responsible for the formation of the broad lines in AGNs (Murray et al. 1996; Chiang \& Murray 1996) has revealed certain advantages of this model, as well as some outstanding observational and theoretical difficulties (e.g., de Kool \& Begelman 1995).

Models of BLRs involving clouds on purely radial (inward/outward) or Keplerian orbits appear to be inconsistent with recent observations of variability in the broad emission-line profiles of Seyfert galaxies (e.g., Peterson et al. 1993). It is also difficult to understand the absence of angular momentum in the (radially) inflowing clouds. Further difficulties accompany the cloud model itself. Among these are the stability of radiatively accelerating clouds against internal radiation pressure gradients and the accompanying hydrodynamical shear, the cloud confinement mechanism, and the source of BLR gas (e.g., Mathews \& Capriotti 1985). Exceedingly large masses for the central black holes, $\gtrsim 10^{9} M_{\odot}$, are required to account for the observed line wings, especially in the brighter AGNs, if these lines are broadened by cloud motion alone. On the other hand, both cloudy and continuous gaseous disk models for the BLRs possess their own share of unusual requirements. Among these are irradiation of the disk from high altitude, a large radial range to account for the entire line profile, disk warping, inflow rates, and low gas temperatures which may lead to Jeans instabilities in the outer disk and possibly to star formation. Also, disk clouds are expected to be short-lived, being sheared on the orbital timescale.
Emmering, Blandford, \& Shlosman (1992, hereafter EBS) proposed a new interpretation of BLRs. In particular, it was suggested that BLRs in AGNs are associated with hydromagnetic (MHD) winds from dusty molecular accretion disks. In this picture, dense molecular clouds are loaded on the magnetic lines threading the disk and are centrifugally accelerated outward and above the disk by magnetic stress, like beads on a rotating wire. When exposed to the central UV continuum source, the clouds are quickly photoionized and produce emission lines, until the clouds dissipate. The EBS model introduced a self-consistent velocity field above the disk, which combines both rotation and outflow. The accretion disk itself was considered to be opaque and to extend beyond the wind radius. Within this framework, both HILs and LILs originate in the wind, at different latitudes and radii. The EBS model accounts also for a transverse confinement of emitting clouds by an ambient magnetic field (see Rees 1987), thus removing a number of long-standing problems associated with the presence of hot and dense intercloud medium in pressure equilibrium with the clouds (e.g., Fabian et al. 1986; Mathews \& Ferland 1987). Furthermore, EBS found that in order to model the broad-line profiles, the BLR clouds need to originate only over two decades in disk radius, if electron scattering by a hot, $\sim 10^{6} \mathrm{~K}$, gas contributes to the line broadening in the wings. The importance of hydromagnetic winds for AGN unification schemes was further emphasized by Königl \& Kartje (1994), while de Kool \& Begelman (1995) studied implications of magnetized winds for broad absorption-line quasars.

In this paper, we propose to test the MHD model in the best studied case of a variable AGN-the Seyfert 1 galaxy NGC 5548. We model the dynamics and geometry of the BLR in this object, constraining it through the observed variable $\mathrm{C}$ IV emission line, its correlation with changes in the central ionizing continuum, and the response across the $\mathrm{C}$ IV line profile. Based on the modeled response to the observed continuum variations, out best-fit model provides the effective size, the dominant geometry, the emissivity distribution in the velocity space, and makes certain predictions about the response of the C IV emission line in NGC 5548. The underlying dynamical model of the BLR is that of a hydromagnetic wind from an accretion disk. By incorporating a number of additional assumptions outlined below and in $\S 3$, we show that the three-dimensional velocity field in the EBS model having both outflow and rotational components can explain the observed profiles and their variability in this object.

A number of specific issues guided us when applying the EBS model to the BLR in NGC 5548. First, based on the line response to the variable ionizing continuum, the cloud population that is responsible for the $\mathrm{C}$ IV emission must be optically thick with hydrogen column densities in excess of $10^{23} \mathrm{~cm}^{-2}$. This follows from the observed $\mathrm{C}$ IV emission which has responded positively to the continuum change. No negative response, i.e., decrease in the $\mathrm{C}$ IV flux with increase of the central continuum flux, has ever been detected. The prevalence of optically thick clouds in NGC 5548 is also supported by the $\mathrm{He}$ II line emission which originates close to the central source and varies with a substantial amplitude and in near tandem with the central continuum. A direct consequence of large column densities in the BLR clouds is that their C IV emission should be strongly anisotropic (Ferland et al. 1992). Second, the overall 
geometry of the BLR in the EBS model is disklike. Hence a number of optically thick clouds are expected to overlap in a particular direction, causing cloud-cloud obscuration of the central UV continuum source. Continuum optical depth effects must, therefore, be included. Last, the C IV emission line appears to be the dominant and the most uncontaminated line in the broad-line spectra of a large number of AGNs. For this reason we limit our discussion to this line only. Although our analysis is applied directly to the BLR in NGC 5548, it is possible that it has much wider consequences for the unified model of AGNs.

In $\S 2$, we provide a brief summary of the monitoring campaigns of NGC 5548. Section 3 gives a general overview of the EBS model, including the modifications that were introduced and the values of the parameters that were used in the present modeling of the C IV line variation in NGC 5548. In $\S 4$, the results of the model calculations are presented, and $\S 5$ compares the model with the data and makes a number of predictions. Additional comments and conclusions are given in $\S 6$.

\section{OBSERVATIONAL SUMMARY ON NGC 5548}

NGC 5548 is a bright $(V \sim 13.5)$ and variable Seyfert 1 galaxy. Because of these characteristics it has received considerable attention in recent multiwavelength ground and space-based campaigns to monitor its continuum and emission-line variability in the hopes of uncovering the nature of both. Early investigations into the emission-line variability of NGC 5548 include Peterson (1987), Peterson, Korista, \& Cota (1987), Stirpe, de Bruyn, \& van Groningen (1988), Maoz et al. (1990), Peterson et al. (1990), Rosenblatt \& Malkan (1990), Wamsteker et al. (1990), and Koratkar \& Gaskell (1991). While useful results were obtained, these data were generally less than optimal in temporal resolution and sampling in determining the physical parameters governing the broad emission-line gas, such as geometry, kinematics, and emission-line responsivity. Thus ambitious monitoring campaigns were launched. In 1988-1989 the International Ultraviolet Explorer (IUE) observed NGC 5548 every 4 days for 8 months (Clavel et al. 1991, hereafter C91); accompanying the ultraviolet data were groundbased optical data spanning the same time period (Peterson et al. 1991). Reliable lags between the emission lines and the continuum were determined for the first time, and crude velocity integrated transfer functions were derived for several emission lines (Krolik et al. 1991; Horne, Welsh, \& Peterson 1991; Dietrich et al. 1993; Maoz et al. 1993). A still more ambitious monitoring program which combined data from the Hubble Space Telescope (HST), the IUE, and from ground-based observatories was conducted in the spring of 1993 to address questions requiring higher signal-to-noise, spectral, and temporal resolution spectra. In this case NGC 5548 was observed once per day in the ultraviolet for 39 days with the HST and once every other day for 75 days with $I U E$, again with accompanying ground-based optical spectra (Korista et al. 1995, hereafter K95). The highquality HST data will be used to constrain our model, while data from both of these campaigns will be considered in our analysis, below.

The primary results of interest here from these two campaigns were (1) the ionization parameter and density of ionized gas vary across the BLR and, hence, the BLR is stratified in ionization, at least in this object, with the highest ionization lines ( $\mathrm{N} \mathrm{V}$ and $\mathrm{He}$ II) forming mainly near to the source of ionizing photons ( $\sim 2$ light-days) and lines of decreasing ionization forming increasingly farther away; (2) the velocity field of line emitting gas is not purely radial. No unambiguous differential lags have been measured across the $C$ IV $\lambda 1549$ profile. With low signal-to-noise (S/N) and marginal temporal resolution, the IUE spectra of the 1988-1989 campaign do not lend themselves to convincing quantitative profile analyses, while insufficient temporal coverage hamper the analyses of the high temporal resolution, high S/N emission line profiles from the 1993 HST campaign (K95). However, the observed variations in both data sets do suggest that the $\mathrm{C}$ IV profile responds differentially. For example, K95 concluded that there were indications that the wings lead the response of the cores of $\mathrm{C}$ IV, which would imply virial cloud motions. Evidence for the presence of any radial motions have heretofore favored inward flows. Crenshaw \& Blackwell (1990) attributed the redward asymmetric response of the C IV emission-line profile during a dramatic decline in the continuum in 1989 to the presence of inflow. Done \& Krolik (1996) also attributed to a modest gravitational inflow component a similar response in the red wing of $\mathrm{C}$ IV to a dramatic increase in the continuum during the 1993 HST campaign. However, the interpretation of these two isolated cases of redward asymmetric response in C IV (it was not seen in other similar continuum variations) is not clear. Wanders et al. (1995) chose not to interpret it, in the context of the classical inversion of the transfer function equation, and suggested a nonstationary origin of the red wing response. Wanders \& Peterson (1996), in their study of the long-term variations in the $\mathrm{H} \beta$ emission-line profile, concluded that the large-scale profile variations in that line are not driven by shorttimescale reverberation effects. Unfortunately, the nature of broad emission-line profile variability remains unclear.

In spite of this, some statements have been made in the recent literature regarding the nature of the flow of broad emission-line gas. Based on analyses of the K95 spectroscopic data, Done \& Krolik claimed to have excluded several kinematical models, among them the rotating hydromagnetic wind model of EBS, although they soften their claim in scenarios for which the far side of the BLR is obscured by, for example, the putative accretion disk. In view of a prevailing trend in the literature to interpret the observations in terms of inflow, it is our intention here to demonstrate that the outflow picture, based on the EBS model of hydromagnetic wind, makes predictions which are entirely consistent with the observations of the BLR in NGC 5548.

\section{GENERAL ASPECTS OF THE MODEL}

\subsection{Hydromagnetic Flow in the Broad-Line Region}

Using asymptotic solutions of hydromagnetic flows from accretion disks and the line-broadening effect of electron scattering, EBS were able to reproduce typical static UV line profiles for BLRs in AGNs. In this section, the EBS model is further elaborated in order to account for the temporal effects detected in the broad C IV line of NGC 5548. We give only the necessary details of the model and discuss modifications we have introduced. More can be found in EBS.

The EBS model assumes that an outflow originates from an optically thick Keplerian accretion disk of a dusty molecular gas. Thus an observer will see only the nearer 
hemisphere of the BLR. Magnetic field lines thread the disk and are frozen into the ionized gas component there (Blandford \& Payne 1982). Gas is occasionally loaded on the magnetic lines and centrifugally accelerated above and away from the disk. The clouds in this flow are assumed to be exposed to the central UV continuum source and to contribute to the line emission between the polar angles $\psi_{l}$ and $\psi_{u}$ above the plane of the disk. The innermost radius of the hydromagnetic wind in the disk plane, $r_{0, \min }$, is defined by the transition from molecular to ionized disk which exists interior to this point. This radius is established by the sublimation temperature of the dust, $T_{\max } \sim 1800 \mathrm{~K}$ (Phinney 1989), and is given by EBS

$$
r_{0, \min }=6 \times 10^{16} L_{44}^{1 / 2} \zeta^{1 / 2} \phi^{-1 / 2} T_{m 18}^{-2} \mathrm{~cm},
$$

where $T_{m 18} \equiv\left(T_{\max } / 1800 \mathrm{~K}\right) ; L=10^{44} L_{44} \mathrm{ergs} \mathrm{s}^{-1} ; \zeta(\ll 1$ at low latitudes above the disk) is the attenuation factor of UV radiation; and $\phi \sim 0.1-1$ is the mean IR extinction efficiency (e.g., Sanders et al. 1989; Shlosman \& Begelman 1989). Because the characteristic timescale for variability of the central UV-soft-X-ray continuum luminosity $L(t)$ ( $\sim$ tens of days) is much shorter than accretion timescale $\left(>10^{3} \mathrm{yr}\right), r_{0, \min }$ is fixed by the (unknown) historical maximum of the central luminosity and is not expected to respond to shorter timescale continuum variability. In other words, the only way to replenish the dust at some radius in the disk destroyed by a "flash" in $L(t)$ is by moving the dusty gas inward from a larger radius. This can be done only on the accretion timescale - the longest timescale in the problem. Because the historical maximum of $L(t)$ is not known and in compliance with the observed He II response, we fix $r_{0, \min }$ at 1 light-day, taking $\left(L_{44} \zeta / \phi\right)^{1 / 2}=0.045$ in equation (1).

The EBS model provides scaling relationships for the density in the MHD flow, the magnetic pressure, density, mass, and size of individual clouds, with the position in the wind. For example, at the base of the wind, the density is $\propto r^{-b}$, the magnetic energy density $\propto r^{-(b+1)}$, and the mass loss per decade in $r$ is $\propto r^{-(2 b-3) / 2}$, where $b$ is a constant. Dependence of physical parameters on the position along the streamline is more complicated and explained in EBS and Blandford \& Payne (1982).

\subsection{Emission Model of the Broad-Line Region}

Two important modifications have been introduced to the emission-line model used by EBS. First, because clouds are optically thick to the Lyman continuum and sufficiently thick to C Iv line photons (Netzer 1990; Ferland et al. 1992), we introduce a simple emission anisotropy, $A(\omega)$, for this line

$$
A(\omega)= \begin{cases}\cos \omega, & \text { for } 0 \leq \omega<\pi / 2, \\ 0, & \text { for } \pi / 2 \leq \omega \leq \pi,\end{cases}
$$

where $\omega$ is an angle between the direction to central source and the direction to the observer, as viewed from the cloud. Equation (2) means that we ignore emission from the backside of the BLR clouds, which heavily biases the observed line radiation to come from the far side of the BLR (Fig. 1). This simple form accentuates the influence of anisotropy on line formation. Although Ferland et al. calculated front/ back $C$ IV intensity ratio from a cloud being $\sim 3: 1$, possible dust opacity was not taken into account. Given that the dusty molecular disk is the source of the wind, the condi-

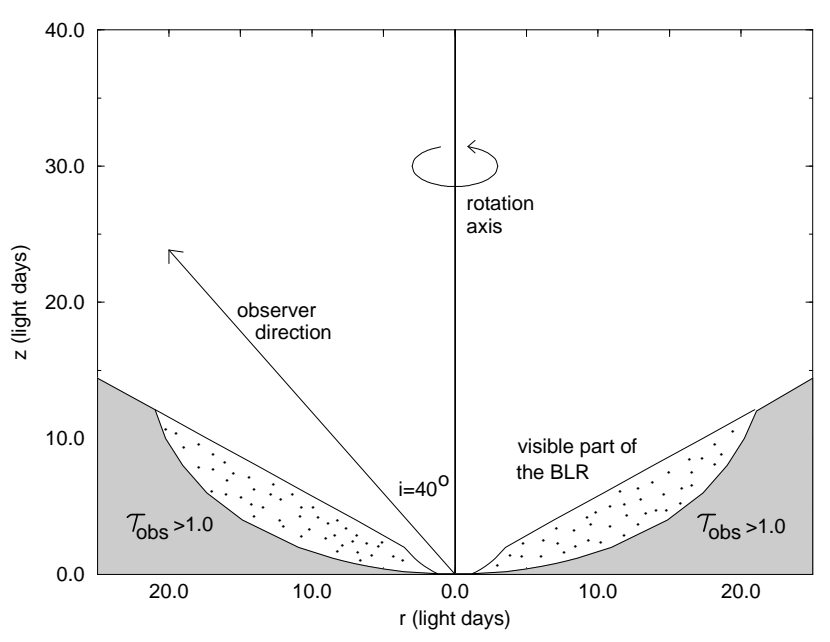

FIG. 1.-Schematic cross-section of the model BLR in the $r z$-plane. The $\mathrm{C}$ IV emission originates between $3^{\circ}$ and $30^{\circ}$ above the disk plane (dotted region). The aspect angle $i$ is the disk inclination to the observer. The gray-shaded region, where $\tau_{\mathrm{obs}}>1$, contains dusty molecular part of the wind.

tions at low latitudes will ensure survival of dust in the neutral parts of the large column density clouds. Consequently, this destruction of $\mathrm{C}$ IV photons will eliminate the $\mathrm{C}$ IV emission from the backside of the cloud.

Second, we use a simple approximation to the continuum optical depth effects in the wind. This is done by assuming that all clouds are optically thick to the ionizing continuum and that foreground clouds shield the background clouds. For the sake of simplicity, we neglect the continuum radiative transfer through the clouds, taking them to be of an infinite optical depth at all energies relevant to $C$ IV. This is identical to having a pure geometrical obscuration. The cloud obscuration is defined as

$$
\tau_{\mathrm{obs}}=\int \sigma_{c} n_{c} d R
$$

where the integral is taken along a spherical radius-vector $\boldsymbol{R}$, starting at the innermost wind boundary $r_{\min }$ at a height $z$ above the disk plane; $\sigma_{c}$ and $n_{c}$ are the cloud cross section and volume number density, respectively. Note, that in our notation, capital $R$ is used for the spherical geometry, while small $r$ denotes a cylindrical radius. Equation (3) can be evaluated analytically using variables of the self-similar MHD wind model:

$$
\tau_{\mathrm{obs}}=3 \gamma F(\psi) R_{0, \min }\left[\left(R / R_{0, \min }\right)^{1 / 3}-1\right],
$$

where $R_{0, \min }$ is the spherical radial coordinate of the innermost obscuring clouds on the ray $\boldsymbol{R} ; \psi$ is defined as an angle between $\boldsymbol{R}$ and the disk surface; $F(\psi)$ is an auxiliary dimensionless function (Fig. 2) which provides the polar dependence of the MHD model. $\gamma=0.29$ lt-day $^{-1}$ is a normalization constant that sets the strength of the cloudcloud obscuration effect in the BLR. The choice of $\gamma$ determines the size of the emitting BLR and comes about from fitting the model $\mathrm{C}$ IV line profile to the data (see $\S 4$ ). The value of $\gamma$ chosen in this paper bounds the C IV emitting region to be within 24 light-days of the continuum source. Clouds lying at $\tau_{\text {obs }} \geq 1$ are completely obscured from the ionizing UV continuum and, therefore, do not emit $C$ IV line radiation. We do expect that hard X-rays will penetrate well beyond this boundary and hence the $\tau_{\text {obs }} \geq 1$ region might 


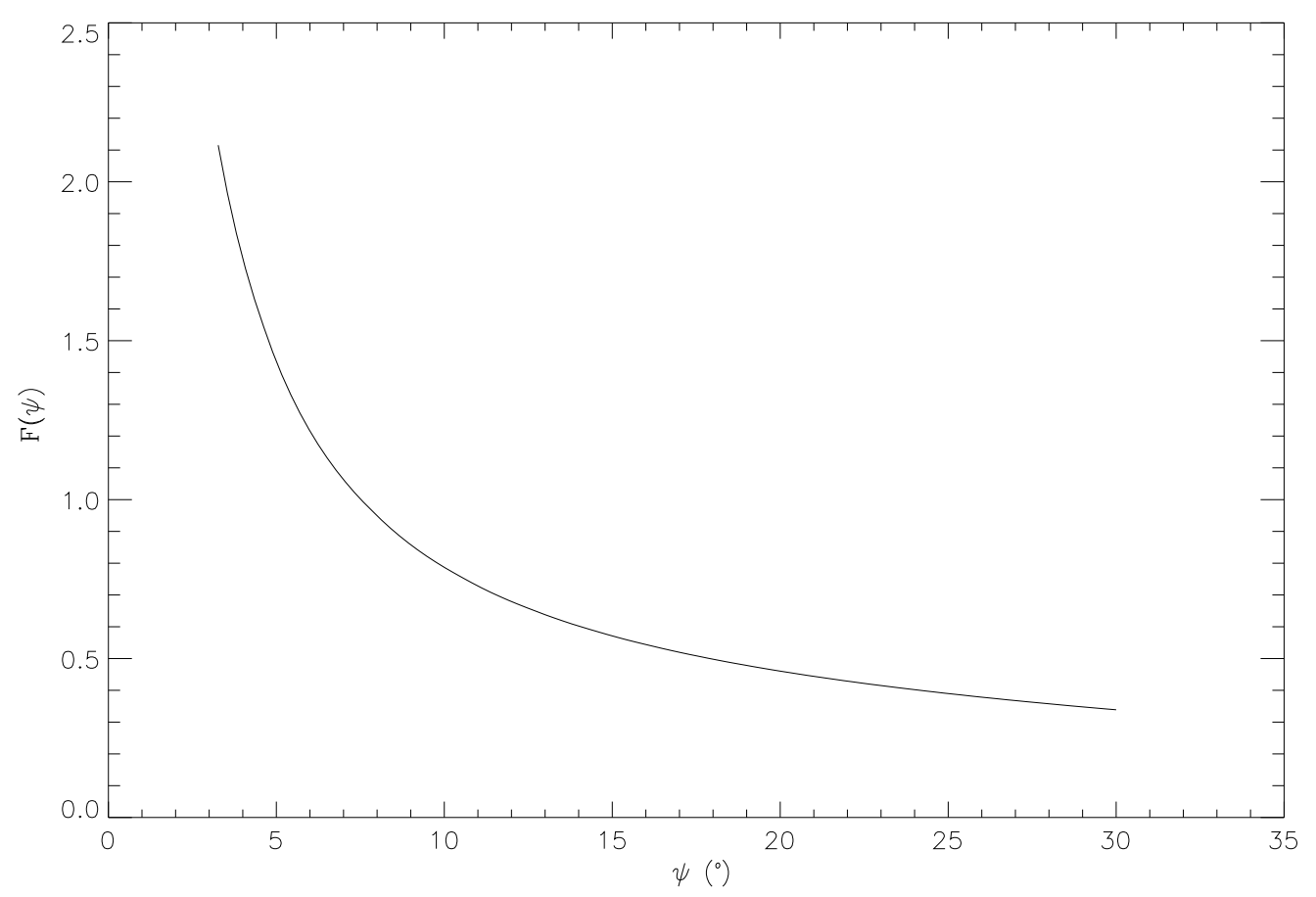

FIG. 2.-The auxiliary function $F(\psi)$ represents the dependence of $\tau_{\text {obs }}$ on the polar angle $\psi$ through $\sigma_{c}$ and $n_{c}$ (eq. [3]). It is obtained by solving equations of the MHD flow (EBS).

be responsible for LILs formation. Cloud obscuration can further enhance the ionization stratification in the BLR.

The C IV volume emissivity of the wind in the direction of the observer is then given approximately by

$$
\eta= \begin{cases}\epsilon n_{c} \sigma_{c} A(\omega)\left(1-\tau_{\mathrm{obs}}\right), & \text { for } \tau_{\mathrm{obs}} \leq 1, \\ 0, & \text { for } \tau_{\mathrm{obs}}>1,\end{cases}
$$

where $\epsilon$ is the surface C IV emissivity of a cloud. The quantity $\sigma_{c}\left(1-\tau_{\mathrm{obs}}\right)$ then represents the area of a cloud that is exposed to continuum radiation and responds by emitting $\epsilon$.

Based on the cloud parameters provided by the EBS model, one can, in principle, calculate a self-consistent $\mathrm{C}$ IV volume emissivity distribution in space. However, such a calculation is impractical, because $\epsilon$ is a function of the incident continuum flux, the cloud column density, and the gas particle density in the cloud. Constraining the model parameters by using the $\mathrm{C}$ IV line profiles then would involve iterations in the multidimensional parameter phase space. Instead, the cloud surface emissivity at a given ionizing flux, $\Phi(\mathrm{H})$, is taken to be a weighted mean of surface emissivities of optically thick clouds distributed uniformly in $\log n(\mathrm{H})$, as explained below. Here $\Phi(\mathrm{H})$ is the photon number flux above 1 Ryd which is incident on the cloud and $n(\mathrm{H})$ is the hydrogen number density of a cloud. The calculations were performed using the photoionization code CLOUDY (Ferland 1994) and the radiative transfer was terminated when the electron fraction fell below 0.1. In this picture, the cloud parameters are not finely tuned; rather a mixture of clouds with a wide range of physical characteristics is assumed to exist at each $R$, subject to selection effects described in the "locally optimally emitting cloud" model (Baldwin et al. 1995).

The cloud hydrogen densities used in CLOUDY calculations ranged over six orders of magnitude, i.e., $10^{8}-10^{14}$ $\mathrm{cm}^{-3}$, and their column densities ranged over $10^{20}-10^{24}$ $\mathrm{cm}^{-2}$. This amalgam of cloud properties is consistent with those prescribed by the EBS model. An analytical fit to this surface emissivity is

$$
\log \epsilon(\mathrm{C} \mathrm{IV})=1.044\left[\log \Phi_{18}(\mathrm{H})\right]^{0.67}+8.0
$$

for $1.0<\log \Phi_{18}(\mathrm{H})<5.0$, where $\Phi_{18}(\mathrm{H})=\Phi(\mathrm{H}) / 10^{18}$ $\mathrm{cm}^{-2} \mathrm{~s}^{-1}$. The shape of the continuum used for the CLOUDY simulations was that of the best fit found by Walter et al. (1994) to IUE and ROSAT data of NGC 5548. We scaled the variations in luminosity to the variations of continuum window observations of the IUE and HST reported in C91 and K95. The luminosity was normalized by the estimated bolometric luminosity of $2.69 \times 10^{44} \mathrm{ergs}$ $\mathrm{s}^{-1}$ assigned to JD 2,449,116, using Hubble constant $H_{0}=$ $75 \mathrm{~km} \mathrm{~s}^{-1} \mathrm{Mpc}^{-1}$ and deceleration parameter $q_{0}=0.5$. This results in a photon number flux of

$$
\Phi_{18}(\mathrm{H})=\frac{2.139 \times 10^{3}}{R_{16}^{2}} f_{\lambda}(t) \mathrm{cm}^{-2} \mathrm{~s}^{-1},
$$

where $R_{16}=\left(R / 10^{16} \mathrm{~cm}\right)$, and $f_{\lambda}(t)$ is the normalized continuum window energy flux at a Julian Day $t$ in ergs $\mathrm{cm}^{-2} \mathrm{~s}^{-1}$. We normalized $f_{\lambda}(t)$ by the $1460 \AA$ continuum window energy flux of $3.30 \times 10^{-14} \mathrm{ergs} \mathrm{cm}^{-2} \mathrm{~s}^{-1}$ on JD $2,449,116$ for the K95 campaign, and by the $1350 \AA$ window energy flux of $3.62 \times 10^{-14} \mathrm{ergs} \mathrm{cm}^{-2} \mathrm{~s}^{-1}$ for the C91 campaign. The line flux contribution at each frequency can be calculated following the prescription given in $\S 4$ of EBS, correcting for anisotropy of line emission and for finite continuum optical depth effects. Because the BLR clouds move nonrelativistically, we limit the accuracy of our calculations to the first order in $v / c$.

To explain the broad emission line profiles, the EBS model invoked both Doppler broadening, arising from the motion of individual clouds in the wind, and electron scattering by a hot gas. No assumption about the pressure equilibrium between the cold clouds and hot electrons was 
made whatsoever. Within this framework, the line cores are formed by unscattered line photons, while the line wings result from electron scattering by a gas at $\sim 10^{6} \mathrm{~K}$. Two models for hot gas distribution have been considered by EBS, i.e., an accretion disk corona (so-called nonlocal scattering model) and an in situ gas surrounding emission clouds (local model). We use the latter model (described in $\S 5.4$ of EBS) for our calculations as it is simpler and its physics is more transparent. EBS have shown that the gas at this range of temperatures is thermally stable in the presence of a confining magnetic field.

\subsection{Building the Model}

A list of model parameters is provided in Table 1A with the adopted values. In addition to the parameters listed in Table 1A are the following which are used to generate model C IV emission-line profiles: (1) the UV-continuum light curve which drives the model $\mathrm{C}$ IV response, (2) a constant narrow $\mathrm{C}$ IV emission-line component.

The model was constrained by the high-quality $\mathrm{C}$ IV emission-line profiles from the HST campaign. To generate these time-variable model emission-line profiles, the $1460 \AA$ continuum window light curve from the $H S T$ portion of the K95 campaign was used. To cover the time period preceding the HST observations, we used a simple functional fit to the IUE continuum. These data were handled by piecewise linear interpolation between data points. The input continuum is shown in Figure $3 a$. We also generated synthetic data using the 1350 A continuum window of the 1988-1989 IUE campaign (C91). In this case, piecewise linear interpolation was also used (Fig. $3 b$ ).

$\mathrm{C}$ IV and other high-ionization lines of NGC 5548 consist of a broad (FWHM $\sim 5000 \mathrm{~km} \mathrm{~s}^{-1}$ ), time-variable component and a time-stationary component with FWHM $\sim 1000 \mathrm{~km} \mathrm{~s}^{-1}$ that rides on top of it. The region that produces the varying broad component is the BLR and the stationary part is formed in the narrow-line region (NLR). We add a stationary NLR component to the synthetic line

TABLE 1A

\begin{tabular}{lc}
\multicolumn{2}{c}{ Model PaRAMETERS } \\
\hline \hline Parameter & Value \\
\hline$\theta_{0} \ldots \ldots \ldots$. & $20^{\circ}$ \\
$\lambda \ldots \ldots \ldots \ldots$ & 5 \\
$b \ldots \ldots \ldots \ldots$ & 1.5 \\
$\alpha \ldots \ldots \ldots \ldots$ & 2.5 \\
$T_{e} \ldots \ldots \ldots$ & $10^{6} \mathrm{~K}$ \\
$f_{s} \ldots \ldots \ldots$. & 0.45 \\
$i \ldots \ldots \ldots \ldots$ & $40^{\circ}$ \\
$\psi_{l} \ldots \ldots \ldots$. & $3^{\circ}$ \\
$\psi_{u} \ldots \ldots \ldots$ & $30^{\circ}$ \\
$M_{\mathrm{BH}} \ldots \ldots$ & $3 \times 10^{7} M_{\odot}$ \\
\hline
\end{tabular}

TABLE 1B

Definitions of C iv Line Segments in Velocity Space

\begin{tabular}{cc}
\hline \hline Line Segment & $\begin{array}{c}\text { Velocity Interval } \\
\left(\mathrm{km} \mathrm{s}^{-1}\right)\end{array}$ \\
\hline Far-blue wing (FBW) $\ldots \ldots \ldots$ & -9000 to -6000 \\
Mid-blue wing (MBW) $\ldots \ldots .$. & -6000 to -3000 \\
Blue core (BC) $\ldots \ldots \ldots \ldots \ldots$. & -3000 to 0.0 \\
Red core (RC) $\ldots \ldots \ldots \ldots \ldots$. & 0.0 to 3000 \\
Mid-red wing (MRW) $\ldots \ldots \ldots$ & 3000 to 6000 \\
Far-red wing (FRW) $\ldots \ldots \ldots$ & 6000 to 9000 \\
\hline
\end{tabular}

profiles produced by the model. For the best fit, we chose a Gaussian profile with a FWHM $=1300 \mathrm{~km} \mathrm{~s} \mathrm{~s}^{-1}$, $F(C$ IV $)=5.9 \times 10^{-13} \mathrm{ergs} \mathrm{cm}^{-2} \mathrm{~s}^{-1}$, and its peak located at a velocity offset of $180 \mathrm{~km} \mathrm{~s}^{-1}$ blueward of the rest frame of the central continuum source taken at redshift of 0.0174 . The adopted NLR parameters were close to those found from independent measurements of the K95 and Crenshaw et al. (1993) $H S T$ data, namely the $F_{\mathrm{NLR}}(\mathrm{C}$ IV $)=$ $6.62 \times 10^{-13} \mathrm{ergs} \mathrm{cm}^{-2} \mathrm{~s}^{-1}$, the FWHM $=1100 \pm 100 \mathrm{~km}$ $\mathrm{s}^{-1}$, and a peak offset of less than $200 \mathrm{~km} \mathrm{~s}^{-1}$.

\subsection{Constraining the Model}

Since the EBS model employs a self-similar solution to the MHD flow, there is no a priori scale imposed on quantities such as the gas density or the column density of a cloud. In order to model the BLR in NGC 5548, it was necessary to establish an absolute scaling. One option was to normalize the emission model by choosing $n_{c}$ and $\sigma_{c}$ in equation (5) at the innermost cylindrical radius of the BLR, $r_{0, \min }$. However, we have shown in $\S 3.1$ that the model depends on their product rather than on each of these quantities separately. This means that the fundamental parameter of the model is the characteristic differential obscuration $\gamma$ introduced in equation (4) as the cloud-cloud obscuration per unit length. Ideally, specifying $\gamma$ and the distribution of cloud surface emissivities fully constrains the emission model, allowing one to calculate the total C IV line profile from the BLR and to compare it with the observed profile. Unfortunately, uncertainties in the Hubble constant and the distance to NGC 5548, anisotropy in the ionizing continuum, assumptions used in calculating $\epsilon$, etc., all make such a comparison almost useless. Instead, in order to overcome these difficulties, we normalize the modeled C IV profile to the observed one by requiring that the flux between relative velocities $+3000 \mathrm{~km} \mathrm{~s}^{-1}$ and $0 \mathrm{~km} \mathrm{~s}^{-1}$ of the model be equal to the flux over the same relative velocity interval in the data. The reason for this choice is that this velocity interval is free from absorption or contaminating emission. We normalized the model line flux on JD $2,449,121$ which was 5 days after the normalization of the continuum, chosen to match the 4.6 day lag between the $\mathrm{C}$ IV emission line and the 1350 A continuum window to the nearest day (K95). The adjustable parameters of the model were then tuned to give a fit to the rest of the data in the line profile segments of the $H S T$ data (defined in $\S 4.2 .2$ ).

The first step in constraining the model was an inspection of model line profiles superposed on the observed line profiles of C IV in K95. The model line shapes are most affected by the velocity field and the emissivity of the BLR. We have used the basic parameters of the MHD model in EBS in order to constrain the velocity field in the C IV emitting BLR of NGC 5548. The parameters of the EBS models have been explored in searching for the best fit to the $C$ IV line profiles. Adjustments to the EBS model parameters reflect our search for proper boundary conditions for the outflow as well as the uncertainty related to the aspect angle of the external observer. The adopted parameters (Table 1A) are close to the values given in the original EBS paper. The parameters of the MHD model used here are as follows: the initial angle of magnetic field lines with the disk, $\theta_{0}$; the dimensionless total specific angular momentum, $\lambda$, in the MHD flow - a conserved quantity (eq. [3.13] of EBS); the MHD flow density power-law index $b$ and the cloud mass power-law index $\alpha$. The geometrical parameters are 


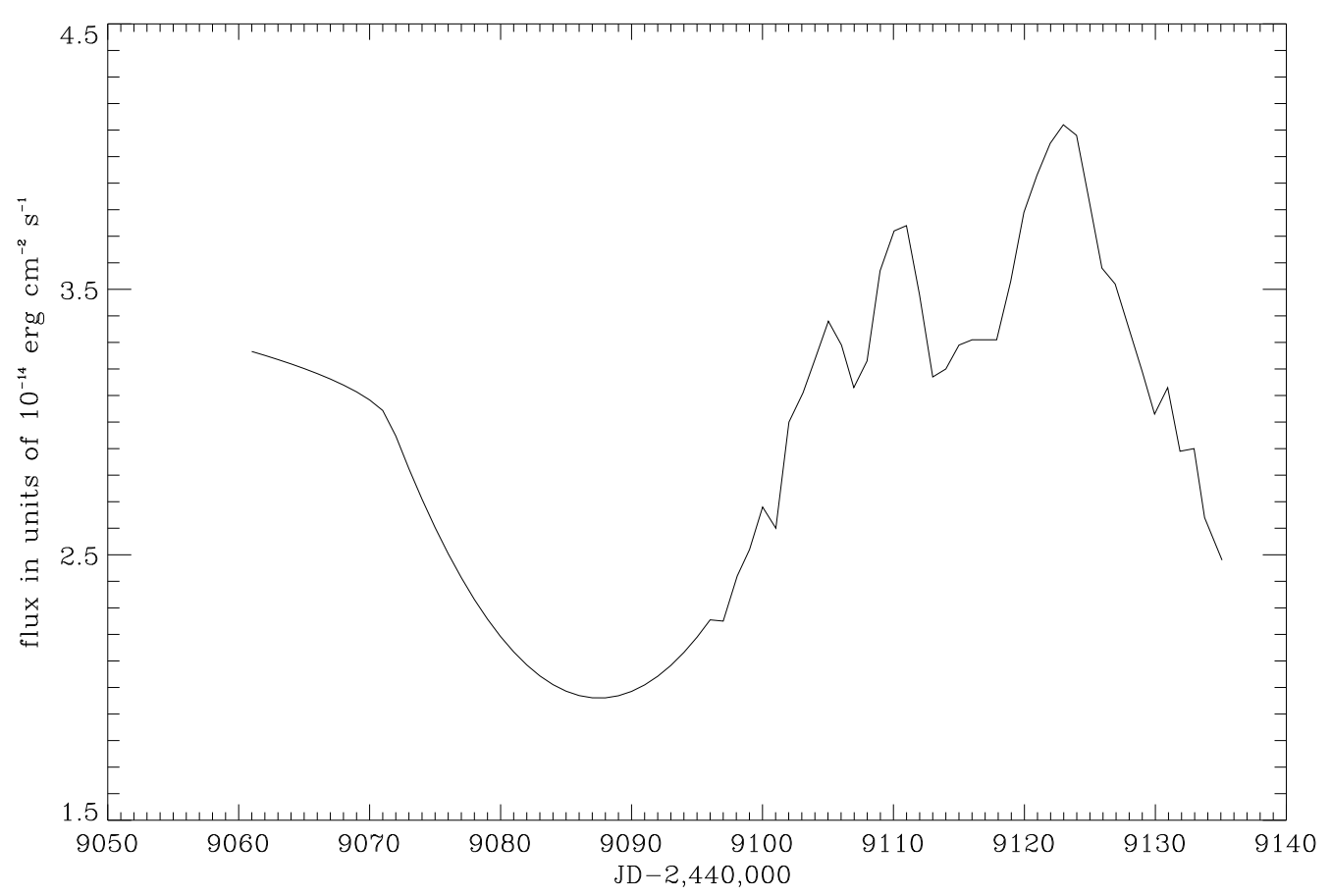

FIG. $3 a$

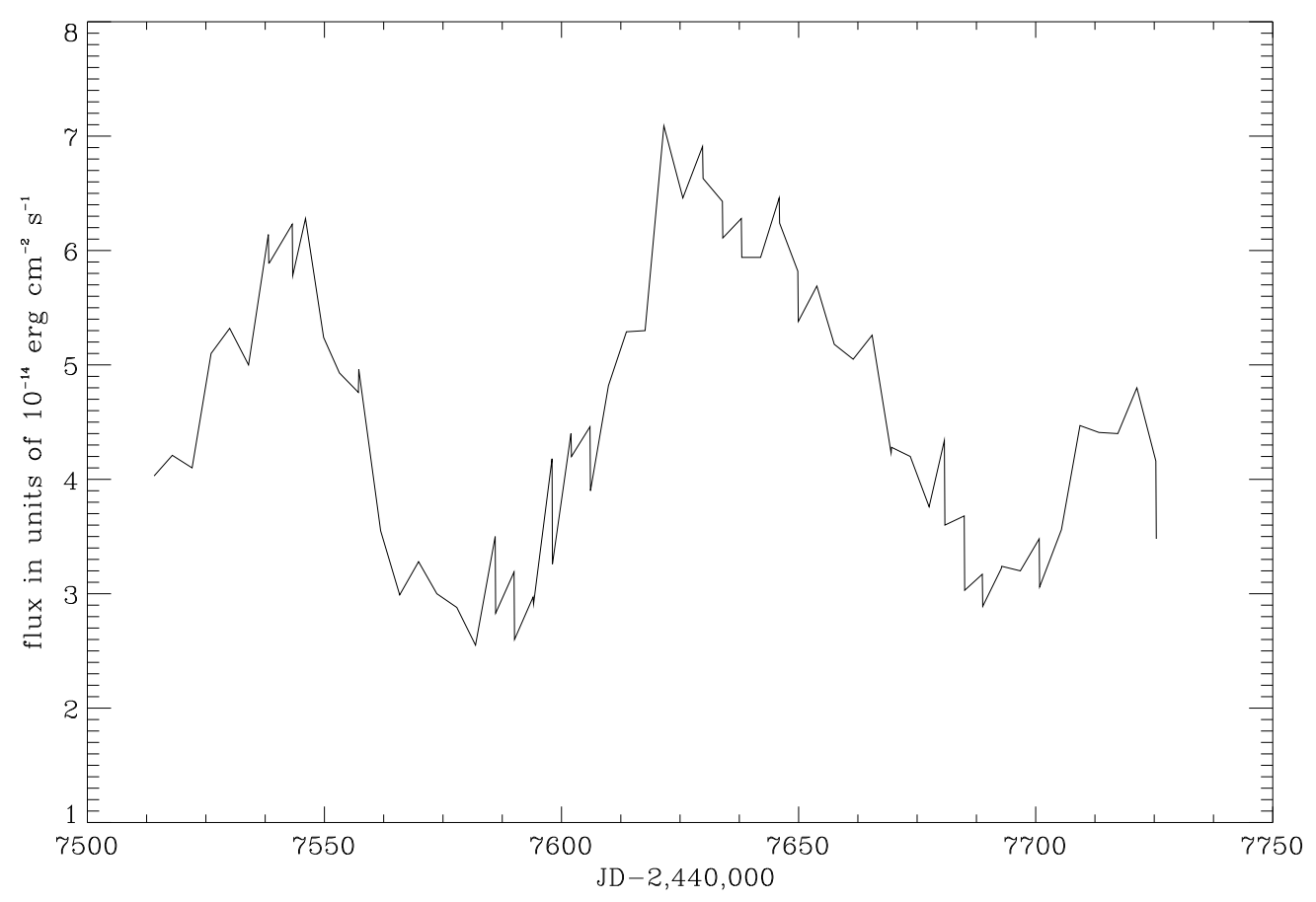

FIG. $3 b$

FIG. 3. - (a) Plot of the 1460 A continuum window flux used to drive the model for the period of the HST portion of the K95 campaign. (b) Plot of the 1350 Å continuum window flux used to drive the model in the C91 campaign.

the aspect angle $i$ of the observer with the rotation axis of the accretion disk; and the upper and lower boundary polar angles of the BLR, $\psi_{l}$ and $\psi_{u}$, as measured from the continuum source. The electron scattering assumes a temperature of $T_{e}$ in the intercloud gas and the fraction of photons that scatter at least once in the intercloud gas is given by $f_{s}$.

Fixing the above parameters of the model allows one to calculate the $\mathrm{C}$ IV line profiles leaving only one degree of freedom in choosing their FWHM. Fitting the observed
$\mathrm{C}$ IV line profiles then constrains the mass of the central black hole, $M_{\mathrm{BH}}$, to be $\sim 3 \times 10^{7} M_{\odot}$. We also note, the physical meaning of $b=1.5$ in the best-fit model is that the mass-loss rate per decade of disk radius $r$ is independent of $r$.

The following features must be accounted for to allow a meaningful comparison between the synthetic and observed C IV lines: (1) several absorption components in the C IV profile, and (2) a small correction in the flux of the extreme 
red wing of $\mathrm{C}$ IV due to contamination by other emission. Corrections for both absorption and contaminating emission to the measurements of the data were based on the differences between the direct integrations and fit to the $\mathrm{C}$ IV emission line (described in $\S 4.2 .2$; see also Fig. 9 of K95).

\section{MODEL RESULTS}

This section is devoted to a direct comparison between the available data on C IV emission line in NGC 5548 and our model for the MHD wind from an accretion disk. Using the observed UV continuum light curve as the driver, we compute the response of the synthetic C IV emission-line profile without varying the model parameters. We compare the observed variable line profiles of K95 with the synthetic line profiles of the model. Finally, based on the crosscorrelations between different segments of the line, we estimate the response times for different parts of the synthetic $\mathrm{C}$ IV emission-line profile and compare to the observed lags.

\subsection{Model C Iv Line Profiles}

Figure 4 provides sample line profiles generated by the model superposed on the HST data profiles. The vertical

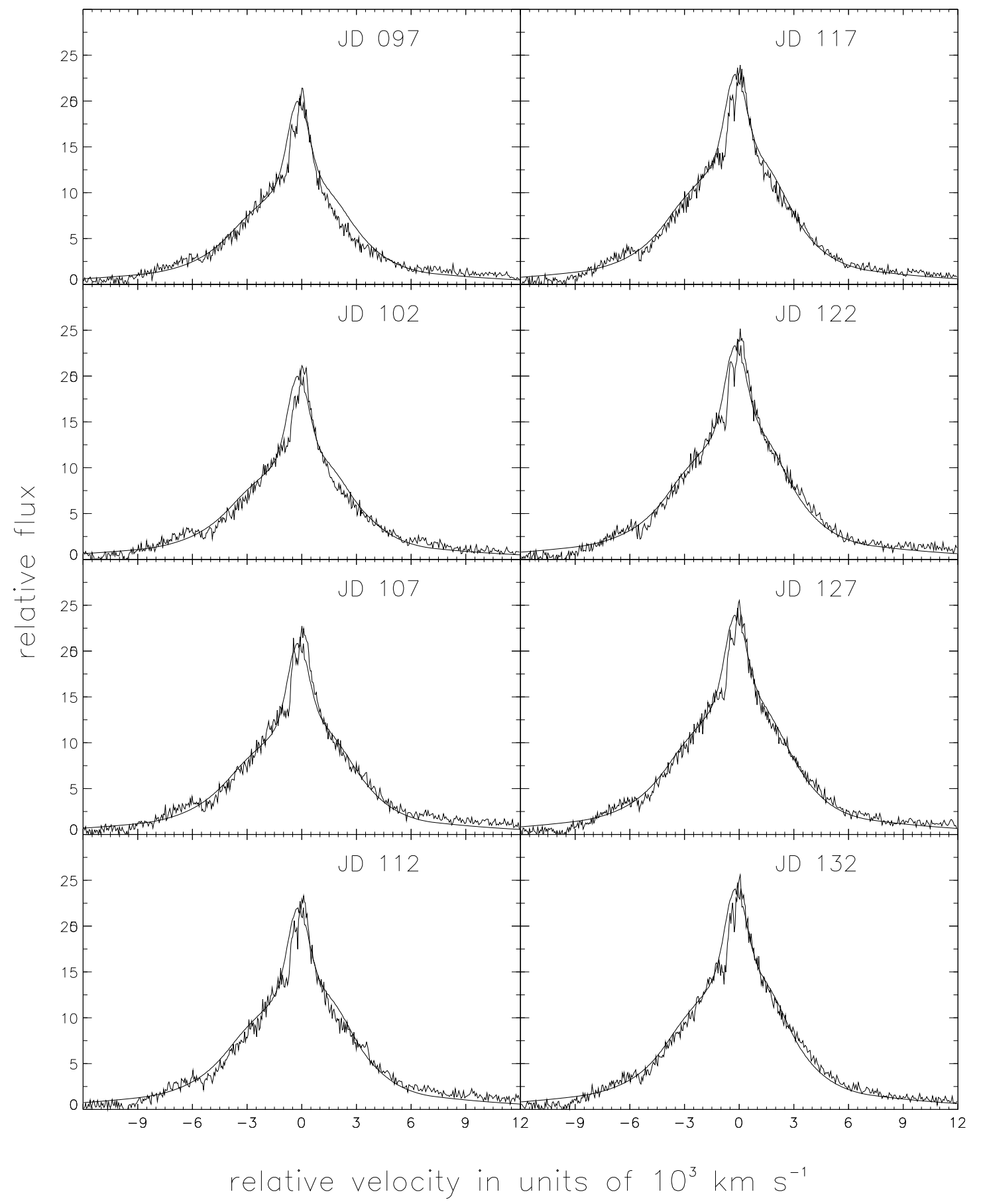

Fig. 4.-A sample of $\mathrm{C}$ Iv line profiles (thick lines) generated by the wind model superposed on the HST data. The Julian dates are shown in the upper right corners. The vertical axis is given in relative intensity units. The horizontal axis gives the Doppler shift in velocity units of $\mathrm{km} \mathrm{s}^{-1}$ with respect to the line center (negative values mean a blueshift). The jagged line represents the observed line profiles. 
axis shows the intensity in arbitrary units and the horizontal axis is scaled in units of relative velocity offset from the center of the $\mathrm{C}$ IV line. The profiles chosen for this figure are at 5 day intervals, starting with JD 2,449,097. A number of distinct absorption features are clearly evident on the blue side of the observed lines which have not been corrected. The line profiles are quite similar in shape. The model initially slightly overpredicts the red flux, but the data and model curves merge together within 10 days. In both campaigns, we find that the synthetic line profiles have a constant blue asymmetry of $\sim 8 \%$ in the wings (using the EBS definition), at $10 \%$ peak line intensity. However, the asymmetry is time-variable in the core. At half-maximum the asymmetry changes from being $5 \%$ red to $2 \%$ blue, during the C91 campaign. During the K95 period, it changes from $0 \%$ to $6 \%$ in the red.

\subsection{Iv Line Light Curves}

In this section we compare the temporal variations in the $\mathrm{C}$ Iv line flux between the model and the data.

\subsubsection{Integrated Line Fluxes}

We first compare the integrated line fluxes for C IV. In Figure 5, the light curve for the integrated line flux for the model is superposed on the data for $H S T$ portion of the K95 campaign. The model curve was created by driving the EBS model with a continuum input scaled by the varia-
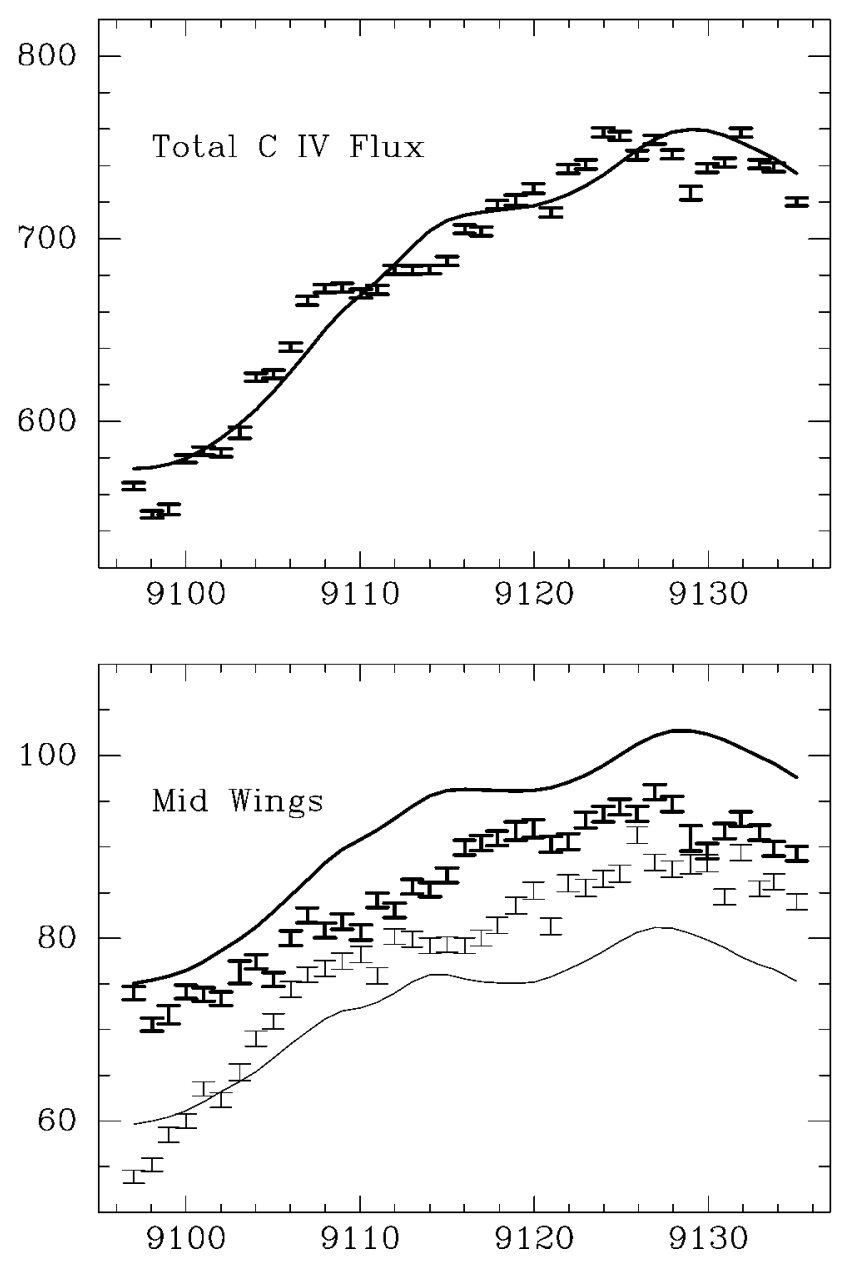

tions of the 1460 Å continuum window as described in $\S 3.2$. In both the data and the model, we have integrated over a velocity interval of $\pm 9000 \mathrm{~km} \mathrm{~s}^{-1}$. This interval was chosen in order to avoid contamination by He II and other emission lines on the red side of the line.

The model follows the general upward trend of the data. The amplitudes are similar and both the model and the data show the same concavity at the beginning and at the end of the time interval, so the large-scale variations are reproduced by the model.

Figure 6 shows a similar plot for the C91 campaign. This time, the model curve was created by driving the EBS model with a continuum input scaled by the variations of the 1350 A continuum window during the C91 campaign. The integrated lines in this figure represent the total flux in the relative velocity interval $\pm 10,840 \mathrm{~km} \mathrm{~s}^{-1}$. The plots in the figure show that the model reproduces three rise and fall events of the data and most of the time the model is within $10 \%$ of the data. This is remarkable because the model was normalized to the absolute value of the flux in the red core on JD 2,449,121 during the K95 campaign and yet provides a reasonable match to the C91 campaign as well, which happened 4 years earlier!

\subsubsection{IV Line Segments}

Looking only at the integrated line flux hides variations that may be occurring in different parts of the line. It is,
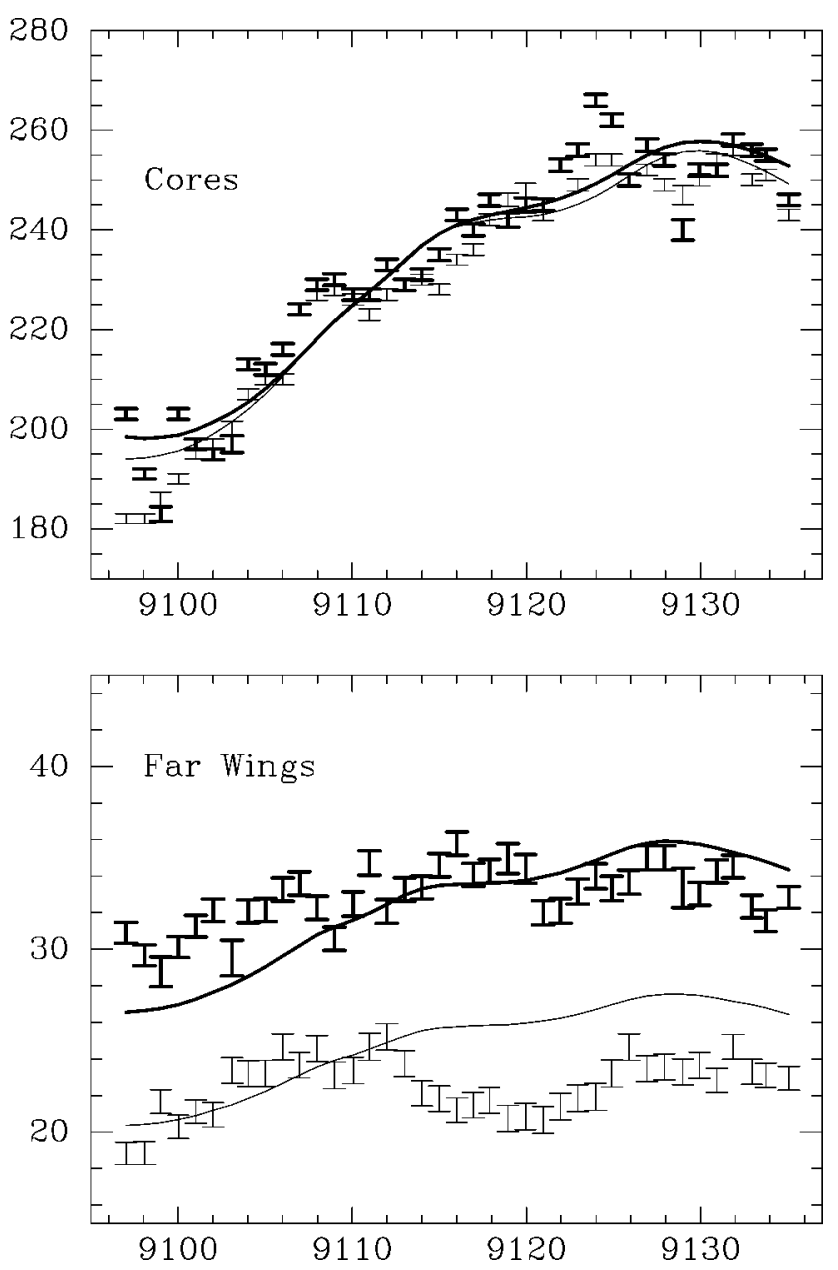

FIG. 5.-Plot of the total line flux and the six line segment fluxes of the data (error bars) and the model (solid lines) as a function of time (JD 2,400,000+) during the HST observing period of the K95 campaign. The velocity ranges for each segment are defined in the Table 1B. The thick error bars and curves corresponds to blue side of the $\mathrm{C} \mathrm{IV} \mathrm{line} \mathrm{and} \mathrm{the} \mathrm{thin} \mathrm{error} \mathrm{bars} \mathrm{and} \mathrm{curves} \mathrm{corresponds} \mathrm{to} \mathrm{red.} \mathrm{The} \mathrm{fluxes} \mathrm{are} \mathrm{normalized} \mathrm{to} \mathrm{units} \mathrm{of} 10^{-14} \mathrm{ergs}^{-2} \mathrm{~s}^{-1}$. 


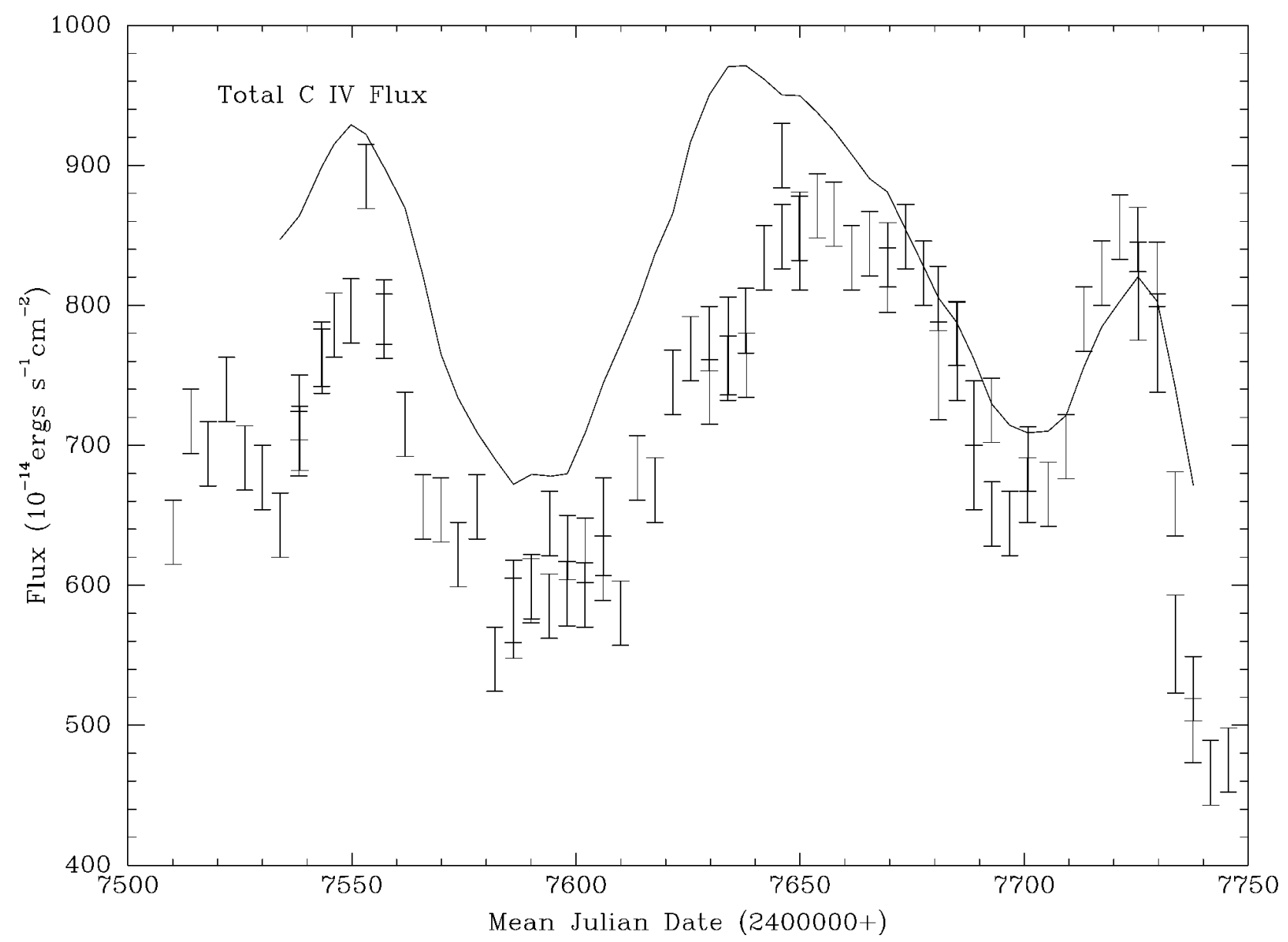

FIG. 6.-Plot of the total $\mathrm{C}$ Iv line flux in the model (line) and the data (error bars) as a function of time during the C91 campaign. The flux is normalized to units of $10^{-14}$ ergs cm $\mathrm{cm}^{-2} \mathrm{~s}^{-1}$. The absence of the synthetic $\mathrm{C}$ IV light curve before JD 2,407,535 is due to the finite time interval (25 days) it takes for the continuum to propagate through the model (continuum data are available only after JD 2,407,510).

therefore, advantageous to dissect the line profile into a number of segments and study each separately. In K95, the $\mathrm{C}$ IV line profile was divided into four distinct segments, i.e., red and blue cores and red and blue wings. We find it more insightful to divide the line into six segments instead. The line segments are defined in Table 1B in terms of Doppler velocity (i.e., relative velocity) as folows, the FBW or farblue wing $\left(-9000 \mathrm{~km} \mathrm{~s}^{-1}\right.$ to $\left.-6000 \mathrm{~km} \mathrm{~s}^{-1}\right)$, the MBW or mid-blue wing $\left(-6000 \mathrm{~km} \mathrm{~s}^{-1}\right.$ to $\left.-3000 \mathrm{~km} \mathrm{~s}^{-1}\right)$, the BC or blue core $\left(-3000 \mathrm{~km} \mathrm{~s}^{-1}\right.$ to $\left.0.0 \mathrm{~km} \mathrm{~s}^{-1}\right)$, the RC or red core $\left(0.0 \mathrm{~km} \mathrm{~s}^{-1}\right.$ to $\left.3000 \mathrm{~km} \mathrm{~s}^{-1}\right)$, the MRW or mid-red wing $\left(3000 \mathrm{~km} \mathrm{~s}^{-1}\right.$ to $\left.6000 \mathrm{~km} \mathrm{~s}^{-1}\right)$, and the FRW or far-red wing $\left(6000 \mathrm{~km} \mathrm{~s}^{-1}\right.$ to $\left.9000 \mathrm{~km} \mathrm{~s}^{-1}\right)$. Note that in this model most of the line emission in the far-wings and some of the emission in the mid-wings consist of photons which have been electron-scattered from the line cores and the midwings, respectively (see $\S \S 3$ and 5).

The flux variability in each of the above segments is shown in Figure 5, where the model curves are superposed on the data. A number of absorption features can be clearly identified in the observed C IV emission-line profile (K95): two weak (Galactic) interstellar absorption systems (C IV $1549 \AA$ and $\mathrm{Si}$ II $1527 \AA$ ) in the blue wing, and at least one semibroad intrinsic system due to $\mathrm{C}$ Iv blueshifted $\sim 700$ $\mathrm{km} \mathrm{s}^{-1}$ relative to the line peak. The data curves have been corrected for absorption and emission contamination in an approximate way. The corrections were based on a com- parison between the direct integration and fits of the line segment light curves presented in K95. The RC and the MRW had no corrections while the BC has been corrected for intrinsic absorption in the same way as in K95. Finally, two-thirds of the K95 correction for the Galactic absorption in the blue wing were applied to the MBW, and the remaining one-third were applied to the FBW.

The FRW was handled differently than the other line segments because it is significantly contaminated with the He II emission which, in comparison to C IV, varies rapidly with time. Fe II is another possible contaminant. It is not possible at this time to estimate what fraction of the original K95 correction between 3000 and $10,840 \mathrm{~km} \mathrm{~s}^{-1}$ should be applied to our FRW, i.e., between 6000 and $9000 \mathrm{~km} \mathrm{~s}^{-1}$, or to estimate its accuracy. Therefore, we simply adjusted the light curve in the data FRW until its mean coincided with that of the model FRW. This amounted to a shift of $-15 \%$. None of the subsequent analysis was based on the FRW.

In the cores, the data RC and $\mathrm{BC}$ have virtually the same amplitude variations, and likewise with the model RC and BC (Fig. 5). In the mid and far-wings, there is a blue asymmetry in both the data and the model line profiles, slightly larger in the latter.

We have made a quantitative comparison of the model line segments with the data line segments by computing the rms fractional deviation for each line segment during the HST portion of the K95 campaign. The rms fractional devi- 
ation, $f$, is defined by

$$
f^{2}=\frac{1}{N} \sum_{i} \frac{\left(X_{i}-M_{i}\right)^{2}}{X_{i}^{2}}
$$

where $X_{i}$ and $M_{i}$ are the integrated line fluxes for the data and the model during the $i$ th observation, and $N$ is the total number of observations. Thus, $f$ is a measure of the magnitude of a typical fractional difference expected between the model and the data at any time. The computed values are as follows, $f_{\mathrm{FRW}}=0.09, f_{\mathrm{MRW}}=0.08, f_{\mathrm{RC}}=0.03, f_{\mathrm{BC}}=0.03$, $f_{\mathrm{MBW}}=0.08$, and $f_{\mathrm{FBW}}=0.07$. All model line segments are within $10 \%$ of the data. The fractional deviation of the integrated line for the entire $\mathrm{K} 95$ is $f_{\text {tot }}=0.02$. We note, that while the model reproduces successfully the general trends of the observed line variations (Fig. 6), the synthetic light curves do not follow the short-timescale, small-amplitude variations. The model limitations are further discussed in $\S 5.5$ ).

Done \& Krolik (1996) measured a $\chi^{2}$ of the deviations about the mean integrated line flux and within each of their integrated velocity bins. Their $\chi^{2}$ value thus assumed that the systematic errors associated with the intrinsic line profiles (e.g., narrow emission-line component, Galactic and intrinsic absorption line systems) were removed upon subtraction of the mean. While this may be true of the Galactic absorption lines and the narrow line component, the intrinsic absorption in the blue core and the contaminating emission likely present in both wings of the line profile are not expected to remain constant over time. A meaningful $\chi^{2}$ of this type must contain knowledge of these systematic errors. Therefore, we chose not to pursue this avenue.

\subsection{Cross-Correlation Analysis of the $\mathrm{C}$ Iv Line}

With a good temporal resolution it is possible to investigate correlations between an emission line (or portions of an emission line) and the continuum. A measure of the correlation is determined by the analysis of the crosscorrelation function, hereafter CCF (e.g., Krolik et al. 1991). This function is defined as

$$
C_{1 \mathrm{c}}(\tau)=\frac{1}{\Delta t \sigma_{l} \sigma_{\text {cont }}} \int\left[F_{l}(t)-\bar{F}_{t}\right]\left[F_{c}(t-\tau)-\bar{F}_{c}\right] d t,
$$

where $\bar{F}_{c}(t), F_{c}$, and $\sigma_{\text {cont }}$ are the continuum light curve, the temporal mean of the continuum light curve, and the standard deviation of the continuum light curve, respectively. $F_{l}(t), \bar{F}_{l}$, and $\sigma_{l}$ are the line emission light curve, the temporal mean of the line emission light curve, and the standard deviation of the emission line light curve. $\Delta t$ is the interval of time during which the continuum and the line are observed. The value of $C_{\mathrm{lc}}$ is the correlation coefficient of the line light curve with the continuum light curve temporally shifted by a time interval $\tau$. For two curves that are well correlated, the correlation coefficient will be close to one and the CCF will have a sharp, well-defined peak. The value of $\tau$, at which the correlation is maximal, is called the response time or lag time of the line with respect to the continuum. Another measure of the response time is the CCF centroid at some fraction of the maximum height of the CCF. The centroid is useful when the peaks of the CCF are rounded, making the identification of the peak difficult. In addition, the centroid of the CCF is mathematically identical to the centroid of another function called the onedimensional transfer function (1DTF). The latter indicates how the line strength changes when the system is driven by a continuum which is a delta function in time. We address the 1DTF later in $\S 5$. In this paper we present both the peak lag and the centroid lag for all reported response times. In practice, the definition of $C_{\mathrm{lc}}(\tau)$ was not used because monitoring campaigns are discrete in time. A discrete analog of $C_{\mathrm{lc}}(\tau)$, as developed by White \& Peterson (1994), is used instead.

The lag time provides an important measure of the size of the BLR by interpreting the lag time as being due to the light travel from a central continuum source to the lineemitting region. The differing lag times for different emission lines have provided the most direct evidence for the BLR being stratified, with the highest ionization lines coming from the innermost regions of the BLR and the lowest coming from the outermost regions. Comparing the lag times of individual segments of a single line with the continuum enables us to study the kinematic structure of the BLR.

\subsubsection{Cross Correlation of Integrated Line Fluxes}

We now compare the results of the CCF lag time calculations for model and data integrated lines in the K95 and the C91 campaigns. As with any numerical calculation involving data, there is an error associated with the uncertainties in the input. The problem in analyzing data lags and centroids is that there is no adequate method in computing the errors (e.g., C91). Only if the peak of the CCF is sharp can the error estimates be made (Gaskell \& Peterson 1987). Errors presented here come from this estimate. More sophisticated estimates of errors based on Monte Carlo simulations also provide an idea of the spread in lag times that will occur, but the results are model-dependent. The errors estimated this way are generally substantially larger than those computed using the Gaskell \& Peterson method, especially when the CCF is not sharply peaked.

The behavior of NGC 5548 during the K95 campaign presents some difficulties. Ideally, it would have been advantageous to use only the high-quality $H S T$ data to perform the CCF peak analysis. To determine a precise lag by utilizing the CCF, however, requires a significant change in the slopes and in the sign of the slopes of the light curves. This happens because, by definition, a CCF becomes constant when either of the light curves is a linear function of time.

During the last 39 days of the K95 campaign, when NGC 5548 was observed with the $H S T$, the C Iv line strength steadily increased. It is far from being clear, however, whether it had peaked by the end of the campaign. In other words, the line curves do not turn significantly downward, as can be seen in Figure 5. The apparent lack of the downturn is problematic because during this time interval the emission light curve is approximately linear, and, as a result the peak of the corresponding CCF is not well defined, if only the HST data is used. To overcome this difficulty, we were forced to augment the HST portion of the K95 campaign with the lower quality integrated line flux of the $I U E$ data. This provided coverage from JD 2,449,060 to JD $2,449,135$. During this time, the line flux went down and then came back up again, so the response was reasonably well sampled. According to K95, four data points were suspect, i.e., JD 2,449,063, JD 2,449,077, JD 2,449,082, and JD 2,449,095, and have been removed from our analysis.

The peak lag and centroids of the model and the data are presented at Table 2. For the K95 campaign, the model 
TABLE 2

Time LAGS FOR INTEGRATED Line: DATA AND Model ${ }^{\mathrm{a}}$

\begin{tabular}{|c|c|c|c|c|}
\hline \multirow[b]{2}{*}{$\begin{array}{l}\text { Time INTERVAL } \\
\text { AND CAMPAIGN }\end{array}$} & \multicolumn{2}{|c|}{ DATA } & \multicolumn{2}{|c|}{ MODEL } \\
\hline & $\begin{array}{l}\Delta t_{\text {Peak }} \\
\text { (days) }\end{array}$ & $\begin{array}{l}\Delta t_{\text {Centroid }}{ }^{\mathrm{b}} \\
\quad \text { (days) }\end{array}$ & $\begin{array}{l}\Delta t_{\text {Peak }} \\
\text { (days) }\end{array}$ & $\begin{array}{l}\Delta t_{\text {Centroid }}^{\mathrm{b}} \\
\quad \text { (days) }\end{array}$ \\
\hline Integrated line (C91) ...... & 8.0 & 11.7 & 7.0 & 8.9 \\
\hline$t_{1}(\mathrm{C} 91) \ldots \ldots \ldots \ldots \ldots \ldots \ldots$ & 8.0 & 9.9 & 9.4 & 9.5 \\
\hline$t_{2}(\mathrm{C} 91) \ldots \ldots \ldots \ldots \ldots \ldots$ & 24.0 & 20.1 & 10.0 & 7.6 \\
\hline$t_{3}(\mathrm{C} 91) \ldots \ldots \ldots \ldots \ldots \ldots \ldots$ & 4.0 & 5.9 & 7.0 & 7.3 \\
\hline Integrated line $(\mathrm{K} 95) \ldots \ldots$ & 4.6 & 7.0 & 8.3 & 9.2 \\
\hline
\end{tabular}

${ }^{a}$ Refer to $\S 4.3 .1$ for a discussion on the uncertainties in the lags.

${ }^{b}$ Centroid measured at the $80 \%$ level.

produced peak and centroid lags of 8.3 and 9.2 days. The Gaskell-Peterson error (hereafter GPE) is estimated at 0.6 days. The data peak lag was found to be 4.6 days with a GPE of 0.6 days; the centroid was 7.0 days. Thus, the model and data centroids are reasonably close to each other, and peaks are within a factor of 2 . However, the adequacy of GPE estimate of an overall error in computing the lag is questionable and the real errors in data CCFs are probably larger, making the differences between the model and data even less significant. The simulations of White \& Peterson (1994) confirm our suspicions that in the case of understanding the response of the emission line, the GPE seriously underestimates the uncertainty in the measured lag.

The C91 campaign involved only IUE data and as a result the uncertainties in the flux values are larger. In addition, the time intervals between successive observations were larger as well. As a result, the GPE for all lag times presented below is between 2 and 3 days. On the other hand, during the C91 campaign, the continuum rose and fell three times and the $\mathrm{C}$ IV line responded with three large amplitude variation events of its own (see Fig. 5). Thus the light variations were well sampled. We computed the lag times, $\tau_{\text {Peak }}$, and centroids, $\tau_{\text {Centroid }}$ for the three separate events and for the entire C91 campaign. The three events were defined by the time intervals: $t_{1}=$ JD $2,447,534.0$ to JD 2,447,581.9, $t_{2}=$ JD 2,447,586.0 to JD 2,447,688.8, and $t_{3}=$ JD 2,447,692.7 to JD 2,447,737.8. The lag ranges for the various time intervals are listed in Table 2, for both the model and the data. The peak lags in the table show that in all three events, the ordering of the peak lag is the same. The shortest is $t_{3}$ followed by $t_{1}$; the longest is $t_{2}$. In events $t_{1}$ and $t_{3}$, the model and data lag values fall within the GPE. Also, the model peak lag determined from the entire C91 campaign is consistent with the data. Because it too falls within the GPE, this suggests that, on the average, the model is consistent with the data on a long-term basis. There is, however, a significant difference between the lag times of the data and the model in the second event. The lag time $t_{2}$ for the data is over twice as long as the model which is the reverse of what was observed in the K95 campaign. In the K95 campaign the line light curve went through a trough, whereas in the C91 campaign event $t_{2}$, the light curve went through a peak. One possible explanation is that, during a sufficiently large increase in the continuum flux, an ionization front pushes its way through the innermost clouds, making these clouds optically thin. The bulk of the emission then shifts to higher radii with longer lag times. The opposite may occur in a low continuum state. Figure $3 b$ shows that the continuum event which gives rise to $t_{2}$ has the highest peak flux and goes through the greatest change in flux. In the K95 data set, the continuum was initially approximately constant, then rapidly dropped and rose again, making the emission light curve form a trough between the IUE/HST campaigns. In principle, given this scenario, the ionization front above the molecular disk would then migrate back toward the continuum source and lag times would become shorter. Since, in our analysis, the clouds are always optically thick, substantial changes in the time lags are not expected with accompanying changes in the continuum.

\subsubsection{Cross Correlation of Synthetic Line Segments}

In this section we present the results of computing the lags for the various line segments of the model for the C91 and the K95 campaigns. We cannot compare these to observations for the C 91 campaign because the individual line segment data are not reliable due to a low S/N. Also, in $\mathrm{K} 95$, the line segment data exist only during the HST portion of the campaign and suffers the same problem as the integrated line does (see above). This means that the error estimates by the Gaskell-Peterson method as well as from Monte Carlo simulations will be of little help. In such a case, only the most general qualitative statements can be made about the actual lags and centroids based upon these data.

In order to demonstrate our point that the data CCFs cannot be utilized for determining peak lags, we show as an example in Figure $7 a$ the CCF of the observed MRW with the continuum. The broad flat top of the data CCF is composed of two shallow bumps. One has a peak at 4 days and another-at 16 days. The bump at 16 days is slightly higher than the one at 4 days, but the height difference is not significant, so there is no clearly defined peak. To an even higher degree, double and multiple peaked flat-top behavior is present in the rest of the data CCFs as well, with the exception of the FBW CCF which shows only one peak at 5 days, but the maximum correlation is weak.

The model lag times and centroids are presented in Table 3A. The first column indicates the wing segment. The next two columns show the lag time and centroid for the K95 campaign. The last two columns show the lag time and centroid for the $\mathrm{C} 91$ campaign. Table $3 \mathrm{~A}$ reveals a sequence of responses of modeled line segments to the underlying continuum variation. The first segment to respond is the MRW, which is followed by the FBW, about a day later. Thus, in the model, the red side of the line responds first. The total sequence, from first to last, is as follows: MRW, MBW, FBW, FRW, RC and BC, meaning that the wings respond first and the cores respond last. Although there is a three-way tie between the FBW, MBW, and FRW in the peak lags of C91, the corresponding centroids follow the time sequence of the modeled response to the K95 continuum.

The main outcome of theoretical analysis of the model is the prediction that different parts of the $\mathrm{C}$ IV emission line respond in a particular order. Though the causal agent of change in the model is the continuum, it is advantageous to compare the relative lags of individual line segments by direct cross-correlation of segment light curves. This accomplishes two goals. First, since noise is associated with the continuum input, the error will propagate into each of the computed time lags. By directly cross-correlating two line segments, we eliminate that source of the noise. Second, it is possible that the two line segments might not correlate 


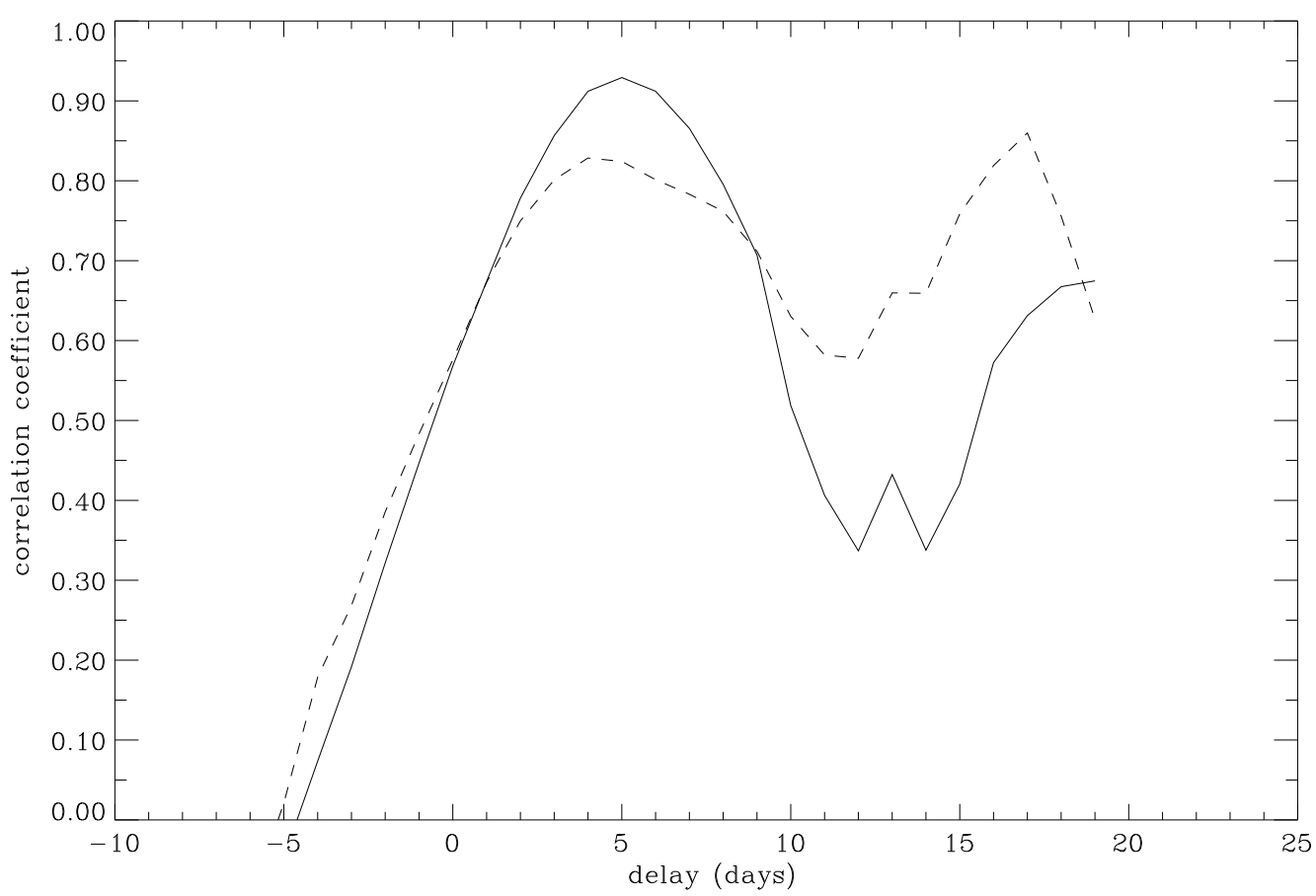

FIG. $7 a$

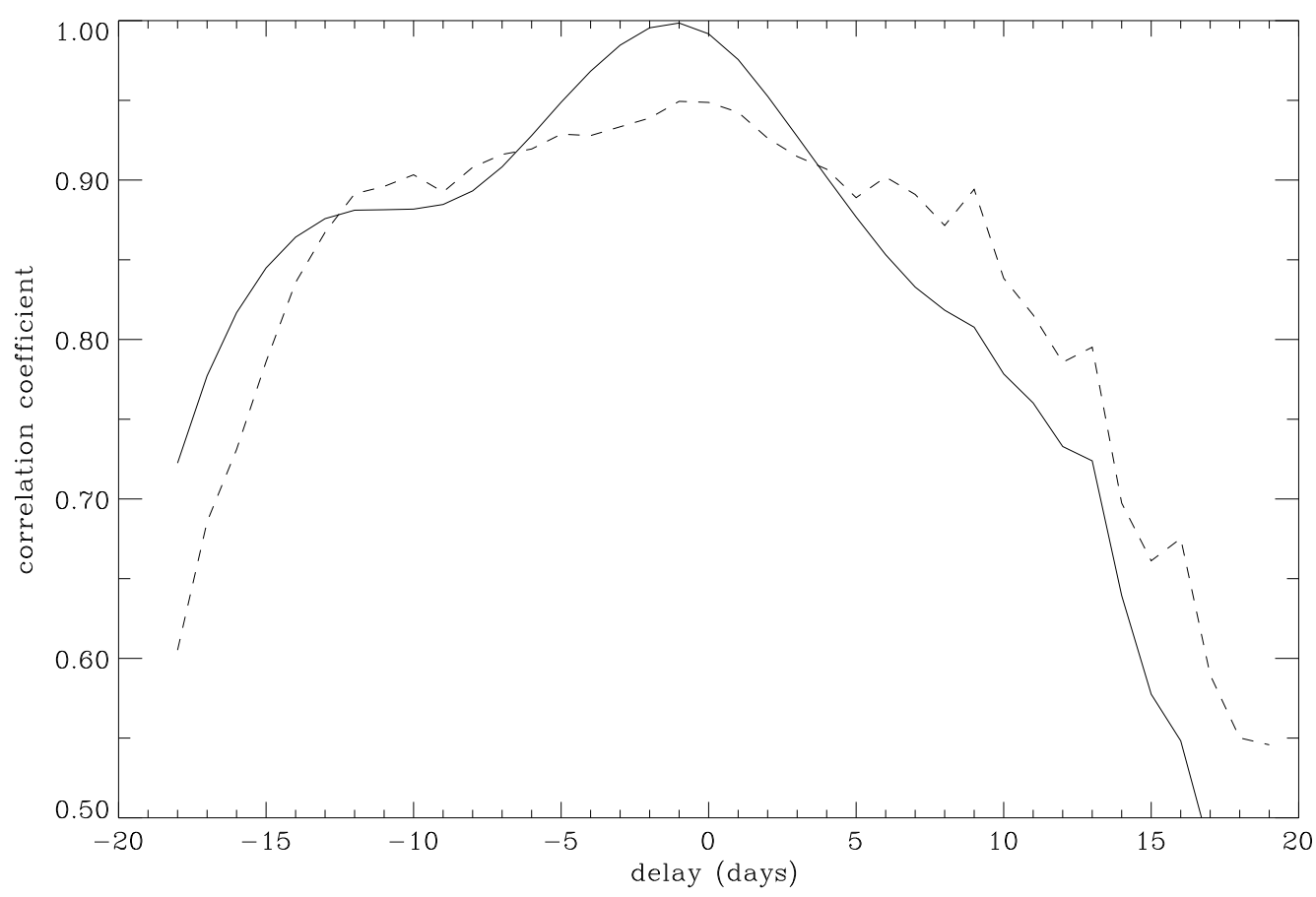

FIG. $7 b$

Fig. 7.-(a) The CCF for the MRW with the K95 continuum. The model CCF is shown as a solid curve and the data CCF is the dashed curve. (b) The CCF for the MBW with the MRW. The model CCF is shown as a solid curve and the data CCF is the dashed curve.

with the continuum as well as they do with one another. In this case, relative time lags can be determined with a greater precision.

Lag times and centroids were calculated for a variety of line segment pairs in the model. The results are presented in Table 3B. In column (1), the segment pairs are listed in a particular order, where the following convention is used. A positive lag indicates that the second entry lags the first. In all seven comparisons of line segments, there is a complete agreement between the lag and the centroid entries of the K95 and C91 campaigns in the ordering of responses.

We note in passing that when segment versus segment cross-correlation is attempted with the available data, the CCFs for line segment pairs show single weak peaks superposed on a wide plateau. Even worse are those pairs that involve the FRW, because it is probably contaminated, and those with the FBW, because its variations have low signalto-noise. An example of MBW versus MRW is shown in 
TABLE 3A

Model Time Lags for C iv Line Segments vs. Continuum

\begin{tabular}{|c|c|c|c|c|}
\hline Line Segment & $\begin{array}{c}\Delta t_{\text {Peak }} \\
\text { (days) } \\
\text { K95 }\end{array}$ & $\begin{array}{c}\Delta t_{\text {Centroid }}{ }^{\mathrm{a}} \\
\text { (days) } \\
\text { K95 }\end{array}$ & $\begin{array}{c}\Delta t_{\text {Peak }} \\
\text { (days) } \\
\text { C91 }\end{array}$ & $\begin{array}{c}\Delta t_{\text {Centroid }}{ }^{\mathrm{a}} \\
\text { (days) } \\
\text { C91 }\end{array}$ \\
\hline FRW ........... & 7.5 & 8.7 & 6.6 & 8.4 \\
\hline MRW ........... & 5.5 & 6.8 & 5.8 & 6.2 \\
\hline $\mathrm{RC} \ldots \ldots \ldots \ldots$ & 8.4 & 9.5 & 9.6 & 9.2 \\
\hline BC $\ldots \ldots \ldots \ldots$ & 9.4 & 10.3 & 9.9 & 10.5 \\
\hline MBW .......... & 6.6 & 8.2 & 6.6 & 7.8 \\
\hline FBW $\ldots \ldots \ldots \ldots$ & 7.4 & 8.5 & 6.6 & 8.2 \\
\hline Integrated....... & 8.3 & 9.2 & 7.0 & 8.9 \\
\hline
\end{tabular}

TABLE 3B

Model Time Lags for C iv Line Segments vs. Line Segments

\begin{tabular}{|c|c|c|c|c|}
\hline $\begin{array}{l}\text { Lines Compared } \\
\text { (1) }\end{array}$ & $\begin{array}{c}\Delta t_{\text {Peak }} \\
\text { (days) } \\
\text { K95 } \\
(2)\end{array}$ & $\begin{array}{c}\Delta t_{\text {Centroid }}{ }^{\mathrm{a}} \\
\text { (days) } \\
\text { K95 } \\
(3)\end{array}$ & $\begin{array}{c}\Delta t_{\text {Peak }} \\
\text { (days) } \\
\text { C91 } \\
(4)\end{array}$ & $\begin{array}{c}\Delta t_{\text {Centroid }}{ }^{\mathrm{a}} \\
\text { (days) } \\
\text { C91 } \\
\text { (5) }\end{array}$ \\
\hline $\mathrm{BC}$ vs. $\mathrm{RC} \ldots \ldots \ldots$ & -0.8 & -0.66 & -0.8 & -1.03 \\
\hline MBW vs. MRW ...... & -1.1 & -1.01 & -1.1 & -1.25 \\
\hline FBW vs. FRW ..... & 0.2 & 0.43 & 0.2 & 0.14 \\
\hline MRW vs. FRW ...... & 1.5 & 2.07 & 1.7 & 2.02 \\
\hline RC vs. MRW ......... & -2.5 & -2.46 & -2.6 & -3.01 \\
\hline FBW vs. MBW ....... & -0.2 & -0.15 & -0.2 & -0.54 \\
\hline MBW vs. BC ......... & 2.2 & 2.58 & 2.3 & 2.67 \\
\hline
\end{tabular}

${ }^{\text {a }}$ Centroid measured at the $80 \%$ level.

Figure $7 b$ which exhibits the best CCF obtained from the data. This particular figure suggests that the MRW responds before the MBW and is consistent with the claim in $\mathrm{K} 95$ that the red wing of C IV responds before the blue wing. Although the far wings respond here between the mid-wings and the cores, we caution that this conclusion is model-dependent and is based on the local electron scattering approximation.

To summarize the most general predictions of the model, (1) the MRW response precedes the responses of all other $\mathrm{C}$ IV line segments; (2) the mid-wings precede the line cores.

\section{DISCUSSION}

The modified EBS model applied to NGC 5548 can successfully reproduce the $\mathrm{C}$ IV line profile variations detected by the HST observational campaign and the line fluxes available from both campaigns. It is important to understand how the geometry and the physics of this model BLR causes the emission-line profiles to change with time. In particular, it must be explained how this model, which has a strong outflow component, is able to produce the shortest lags on the red side of the line. In this section we address these issues and also discuss the limitations of the present model.

\subsection{The Model: Two-Dimensional Transfer Function}

The two-dimensional transfer function (2DTF) provides a means of analyzing the velocity-resolved response of a system to a driving input, at relative velocity $v$ and as a function of time $t$ (Blandford \& McKee 1982; Welsh \& Horne 1991). The 2DTF, $\Psi(v, t)$, is related to the luminosity of the driving continuum $L_{c}(t)$ and the line profile strength
$L_{l}(v, t)$ via

$$
L_{l}(v, t)=\int_{-\infty}^{\infty} d t^{\prime} L_{c}\left(t^{\prime}\right) \Psi\left(v, t-t^{\prime}\right) .
$$

If a delta function continuum pulse is substituted into equation (10), then $L_{l}(v, t)=\Psi(v, t)$. Thus the 2DTF is the response of the system to a delta function pulse. By analyzing the 2DTF we may determine which parts of the line profile respond first, which parts of the profile respond last, and which parts of the profile respond with the greatest strength. The information gained from the 2DTF may then be interpreted in terms of a particular model.

Figure $8 a$ shows the 2DTF for the model. It was obtained by propagating a rectangular 0.1 day continuum pulse. Since the NLR is stationary on the timescales discussed in this paper, it was ignored in calculation of the 2DTF. The outer edge of the C IV emitting region in the model is set by obscuration of the central continuum source. At $30^{\circ}$ above the plane of the disk, along the upper boundary of the BLR, the obscuration effect is the weakest. At this angle, the central source, as viewed from the outflowing clouds, is completely obscured at a distance of 24 light days. At an observer's aspect angle of $40^{\circ}$, a maximal time lag of 28 days for this part of the $\mathrm{C}$ IV emitting region will be measured. The emission flux contribution from the region corresponding to this delay, however, is negligible, so the bulk of the emission corresponds to earlier response times.

As can be seen in Figure 8a, the 2DTF consists of two parts, a strong central region (the 2DTF core) between relative velocities $\pm 6000 \mathrm{~km} \mathrm{~s}^{-1}$, and low flat wings on both sides of the central region. The 2DTF core corresponds to the $\mathrm{C}$ IV line core and the mid-wings. This core grows rapidly for the first 4-5 days. The first response in the 2DTF is observed at around $\pm 7000-7500 \mathrm{~km} \mathrm{~s}^{-1}$. These emission "horns" arise at the contact points of the innermost possible paraboloid of constant light travel time to the observer with the BLR (i.e., corresponding to the minimal lag). In the next section we provide a detailed analysis of the threedimensional velocity field and of cloud emissivity function which fully explain the time-dependent effects of the 2DTF uncovered here.

Both red and blue sides of the 2DTF core rapidly rise, but the red side responds faster and more strongly than the blue side. This can be clearly seen in the contour density in Figure $8 a$. This trend is repeated again during the decline, i.e., the red side of the 2DTF core drops rapidly in comparison with the blue side. The steepest decline occurs in the MRW. From MBW to BC to RC, the decline becomes more shallow. The difference in the amplitude between the red and blue sides of the 2DTF core at early times is roughly $30 \%$. As we show in $\S 5.2$, this is due to excess of emitting gas with redshifted velocities at small $r$ over that with blueshifted velocities.

In the region of the low flat wings of the $2 \mathrm{DTF}$, the emission grows in concert with its core, staying always low. The contour levels in the far wings, as exhibited by Figure $8 a$, do not exceed the $10 \%$ level. Most of the emission in the far wings is due to electron scattering of the line core photons. Comparison of Figures $8 a$ and $8 b$ shows that the model 2DTF far wings are considerably reduced in the absence of scattering. This and the strength of the line core explain why they respond similarly to the line core.

The local scattering model for the far wing origin implemented in this work has an important advantage of being 


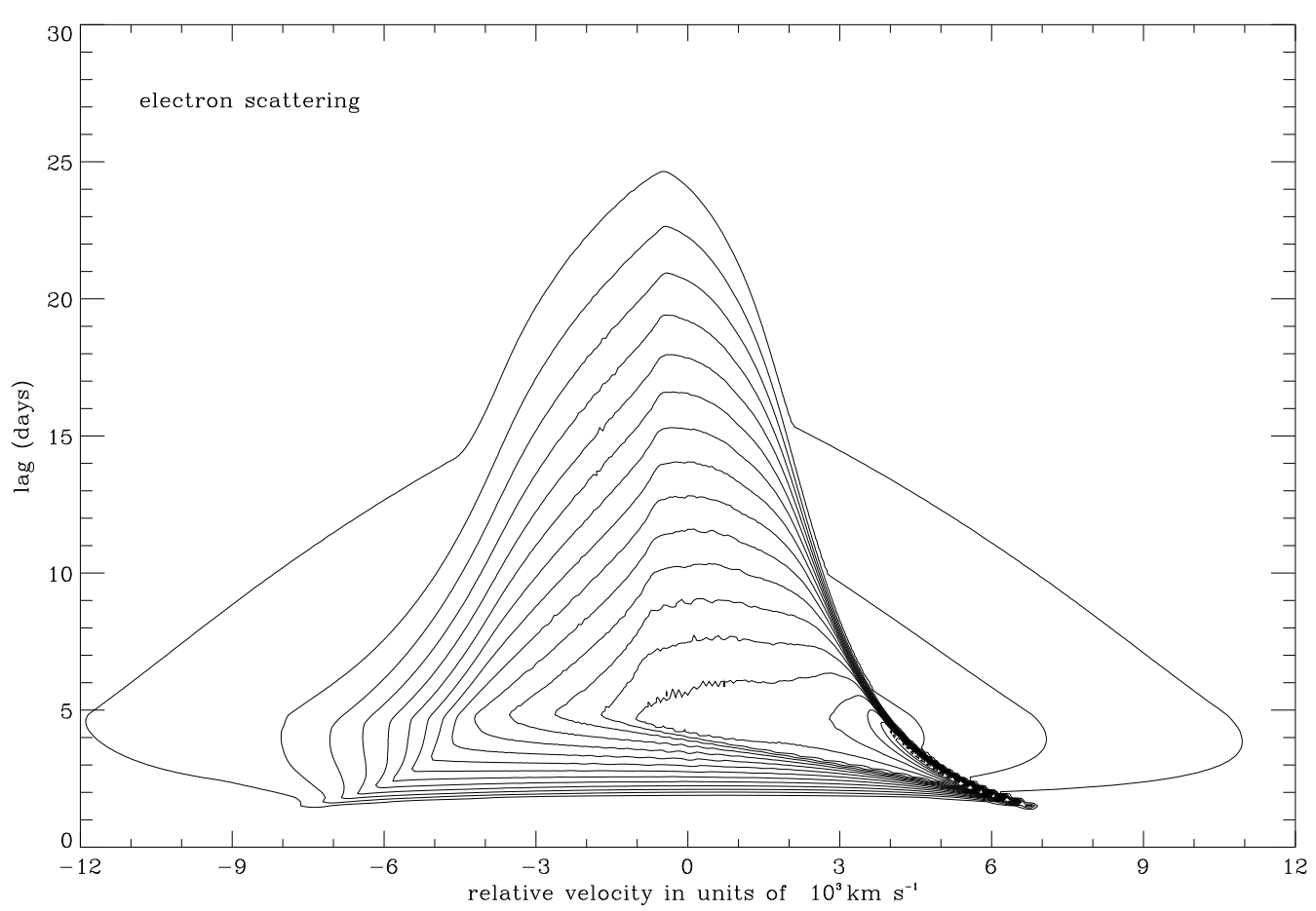

FIG. $8 a$

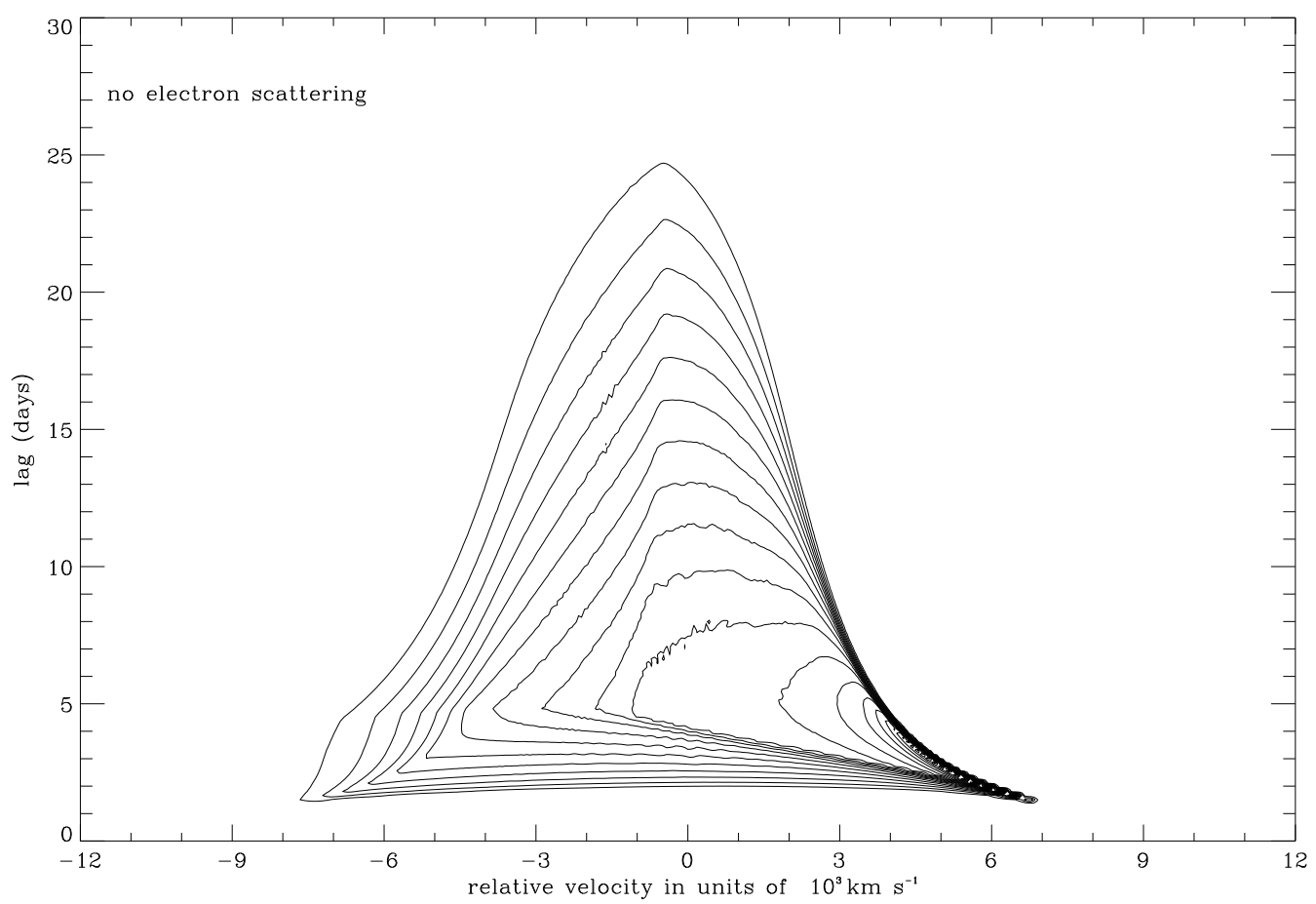

FIG. $8 b$

FIG. 8. - (a) The 2DTF, $\Psi(v, t)$, for the model driven by a rectangular 0.1 day pulse and in the presence of local electron scattering. The contour levels differ by $5 \%$. (b) The 2DTF, $\Psi(v, t)$, for the model driven by a rectangular 0.1 day pulse, without electron scattering. The contour levels differ by $5 \%$.

conceptually and technically simple. However, it may be an oversimplification. The nonlocal scattering model used in EBS, by calculating the far-wing contribution from the disk corona, is probably more realistic, especially because evidence points toward the Balmer lines far-wings being stationary in time in at least one case of Mrk 590 (Ferland, Korista, \& Peterson 1991; Peterson et al. 1993). Within the nonlocal scattering model, the far-wings are formed at various radii from the central source, hence their response to continuum variability should be nearly obliterated.

\subsection{The Model: Projected Velocity Maps}

The velocity field of the EBS flow is a mix of rotation and outflow. At the lower boundary of the BLR, it is predominantly Keplerian, gradually making transition to outflow as the altitude above the disk plane is increased. To under- 
stand the disposition and response of material emitting at a particular velocity, we dissect the model BLR by cutting it with planes parallel to the accretion disk at different $z$. We then map out regions in each plane which correspond to the (line-of-sight) velocity intervals of the line segments, from the FRW to the FBW. This enables us to follow the changes in the spatial distribution of velocity-bounded intervals as the altitude is increased.

In the model presented in this paper, an observer views the BLR looking down at the disk, at an aspect angle of $40^{\circ}$ with the $z$-axis (Fig. 1). In the absence of electron scattering contribution to the $\mathrm{C}$ IV line shape, it is possible to identify regions in the BLR which contribute to the particular line segments. In other words, in this case, the shape of the modeled line profile corresponds to the actual distribution of emission in the velocity space of the line-of-sight velocities. Fortunately, as shown by Figures $8 a$ and $8 b$, the core and the mid-wings of $\mathrm{C}$ IV line (or the 2DTF core) have negligible contribution from the electron-scattered light. Therefore, the behavior of the $\mathrm{C}$ IV core and mid-wings (or the 2DTF core) can be understood by analyzing the spatial distribution of relative velocities in the BLR model. At the same time, the far-wings of the $\mathrm{C}$ IV line (and the wings of the 2DTF) are heavily dominated by electron scattering, which makes them unsuitable for this type of analysis. In this section, we analyze the $C$ IV emission distribution based on the three-dimensional kinematics of the outflow from the disk neglecting the contribution from the electron scattering. Only unscattered light in the FRW and the FBW is accounted for.

Figure 9 presents eight two-dimensional cross sections of the BLR at different altitudes above the disk plane. The vertical axis is the cylindrical radius, $r$, measured in light days. The horizontal axis is the azimuthal angle measured in degrees. The gray scale in Figure 9 demarks the regions which have line-of-sight velocities that correspond to the velocity intervals of the six line segments defined in $\S$ 4.2.2. The shades of gray, from darkest to lightest, correspond to the FRW-to-FBW sequence. The redshifted rotational velocities are between $90^{\circ}$ and $180^{\circ}$, and the blueshifted ones between $180^{\circ}$ and $270^{\circ}$. All areas shaded black in Figure 9 represent spatial regions which do not contribute any emission to the line profile because of the cloud emission anisotropy. The observer is at an azimuthal angle of $0^{\circ}$. Angles smaller than $90^{\circ}$ and larger than $270^{\circ}$ are not shown, as they would be shaded entirely black, because this part of the BLR is not observable. The two horizontal lines in Figures $9 a-9 h$ provide the spatial extention (the inner and outer cylindrical boundaries) of the emitting BLR at each altitude. The observed $\mathrm{C}$ IV emission is strictly limited to lie within these boundaries. The cross-hatched region above the upper horizontal line represents a region of the outflow that does not emit $\mathrm{C}$ IV because of the cloud obscuration, here $\tau_{\mathrm{obs}}>1$. The cross-hatched region below the lower horizontal line is overionized, i.e., it lies within the inner boundary of the BLR, and no C IV photons are generated here. Because Figure 9 accounts for the unscattered emission component only, it must be considered in conjunction with Figure $8 b$ (the unscattered 2DTF).

Inspection of Figures 1 and 9 reveals that the $C$ IV emitting regions at higher $z$ cross sections are also located at greater distances from the continuum source than the lower ones. This means that the possible response times for an emitting cloud become longer as the altitude of the cross section is increased. What complicates the visualization of the three-dimensional velocity field is that there are two components to the outflow velocity which must be accounted for, i.e., along the $z$ and $r$-axes.

Close to the disk surface, the velocity field is dominated by Keplerian rotation with a small addition of $r$ and $z$ components of the outflow (Fig. 9a). Rotation is responsible for the overall symmetry, as seen from the distribution of gray-shaded areas around the azimuthal angle of $180^{\circ}$, meaning that the line profile must be more or less symmetric in the core. A close inspection of Figure $9 a$ reveals that the area corresponding to the MRW is larger than the area of the MBW. This prevalence of redshifted C IV emission in the mid-wing is the signature of the $r$-component of the outflow. The clouds that contribute to the MRW at this low altitude lie at small $r$ and have maximal allowable lineof-sight velocities directed away from the observer. Their emission contribution is substantial because of two reasons. First, being at small $r$, they are subject to a large incident flux. Second, because of the emission anisotropy, clouds moving away from the observer expose their illuminated surfaces. Thus the redshifted emission is particularly bright in the 2DTF.

The signature of the $z$-component of the outflow velocity at small $r$ (corresponding to small lag times) in the 2DTF can be observed at the first contact points, occurring about 0.1 light days above the disk, near $\pm 7000-7500 \mathrm{~km} \mathrm{~s}^{-1}$ (Fig. $8 b ; \S 5.1$ ). The corresponding emission "horns" in the 2DTF are not symmetrically placed in Figure $8 b$, both being slightly blueshifted compared to their projected Keplerian velocities at the first-contact points. The origin of these "horns" was discussed in $\S 5.1$ in the context of the time lags: these are the contact points (intersections) of the innermost possible paraboloid of constant light travel time to the observer with the $\mathrm{C}$ IV emitting gas. The emission "horns" appear blueshifted because the first contact points are near the disk plane at almost right angles with respect to the observer's line of sight. The projection of the radial outflow component onto the observer's line of sight nearly vanishes here. Thus the blueshifted $z$-component in the outflow is emphasized. The observed emission at the first contact points is small, because the clouds, observed here at almost right angles, have their C IV emissivity heavily suppressed by the emission anisotropy.

As the cross section altitude increases, the velocity field becomes distorted toward the blue (Figs. $9 b-9 h$ ). This is a direct consequence of the growing outflow velocity in the $z$-direction superposed on the rotational velocity component. The $r$-component of the outflow velocity decreases with height. If it were not for the presence of an opaque underlying accretion disk, the emission would be distributed symmetrically in velocity space.

The altitude dependence of the velocity field is seen most clearly in the MRW, which turns into an "island" at 1 light-day above the disk. By 2 light-days above the disk, the MRW has disappeared completely. Thus, of the four velocity intervals that make up the 2DTF core, the response of the MRW is more "tightly bound" than the other line segments (these are still present, even at a cross-section altitude of 8 light-days). Consequently, the MRW responds before the RC, BC, or the MBW. The unscattered FRW and FBW in Figure 9 are bound even more tightly than the MBW, and as a result respond even faster than the MRW. However, their intensity is so low that, when the scattered 

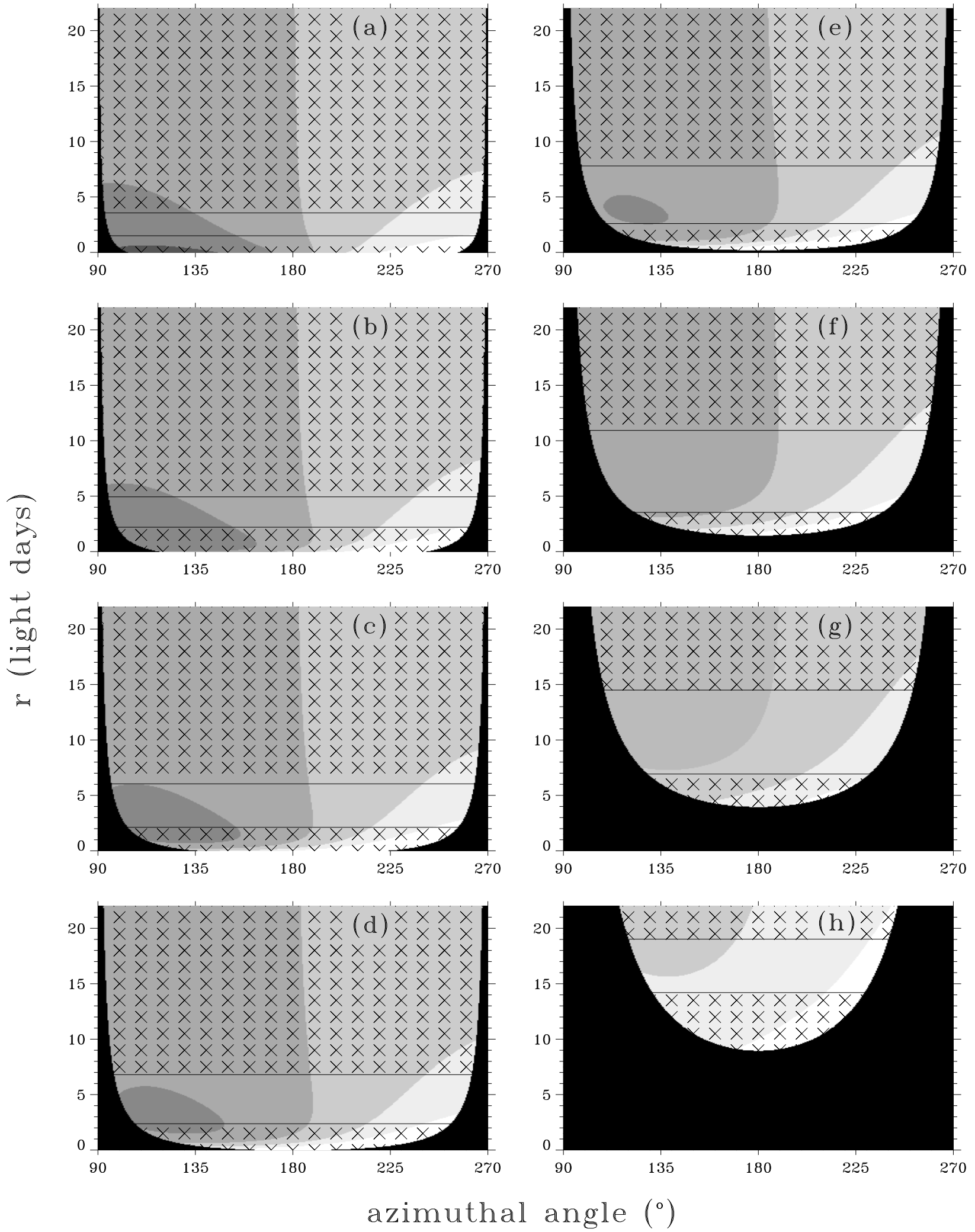

FIG. 9.-Two-dimensional cross sections of the BLR at different increasing altitudes above the disk. $(a)-(h)$ are at $0.2,0.4,0.6,0.8,1.0,2.0,4.0$, and 8.0 light-days above the disk plane. The azimuthal angle in the plane of the disk is limited to $90^{\circ}-270^{\circ}$ because only this part of the BLR is directly observable due to the cloud emission anisotropy. The area between $90^{\circ}$ and $180^{\circ}$ is receding and the area between $180^{\circ}$ and $270^{\circ}$ is approaching with respect to the observer. The black areas are regions which also cannot be seen by the observer because of the cloud emission anisotropy. The dark-to-light gray scale represents the FRW-to-FBW transition (neglecting electron scattering broadening of the line!). The horizontal solid lines represent the inner and outer cylindrical boundaries of the C IV emitting BLR at each altitude. The cross-hatched areas of nonemitting material are left for convenience only. Above the upper horizontal line $\tau_{\text {obs }}>1.0$ while below the lower horizontal line the clouds are overionized. Note that the MRW turns into an "island" and disappears by 2 light-days above the disk (see $\S 5.2$ for additional explanations). 
light is superposed on it, the response is dominated by the (RC and BC) scattered light. Inspection of Figures $8 a$ and $8 b$ shows that the far-wings peak later on, if the electron scattering is present.

As for the remaining three regions which make up the 2DTF core, the RC, BC, and MBW, a migration of the (gray-shaded) boundary between the $\mathrm{RC}$ and the $\mathrm{BC}$ and also the $\mathrm{BC}$ and the MBW occurs as the cross section altitude is increased. In each cross section in Figure 9, the mean radial position of the $\mathrm{RC}$ region is larger than that of the $\mathrm{BC}$, and the mean position of the $\mathrm{BC}$ is larger than that of the MBW. The MBW hence will respond before the BC, and the $\mathrm{BC}$ is expected to respond before the RC. This explains the order of the responses in the 2DTF core, i.e., the MRW responds first followed by the MBW, the $\mathrm{BC}$, and finally the RC.

\subsection{The Model and the Data: One-dimensional Transfer Function}

The one-dimensional transfer function, 1DTF, shows the temporal emission response of the overall BLR. The mean line profile, which is the time average of the line profiles, on the other hand, does not provide timing information on the BLR, but shows the steady state line profile. In this section, we analyze both of these functions calculated by the model, in an effort to compare them with those derived from the data.

We obtain the $1 \mathrm{DTF}$ by integrating the $2 \mathrm{DTF}$ along the line of sight velocity axis (Fig. 10). The resulting 1DTF shows a peak at 4-5 days and trails off to zero intensity at 28 days. The centroid of the model $1 \mathrm{DTF}$ is at 8.6 days.

The behavior of the model 1DTF in Figure 10 can be compared to transfer functions derived from the monitoring of NGC 5548 (Krolik et al. 1991; Peterson 1993; Wanders et al. 1995; Done \& Krolik 1996) by a variety of inversion methods used to extract the 1DTF from the data. In
Wanders et al. (1995), who used the K95 data, the 1DTF declines toward zero intensity at large lags (25 days), similar to our model. At small lags, the data 1DTF is compatible with a wide plateau or even a slight depression within 5 days, although Wanders et al. preferred a monotonically rising function toward the zero lag. Our model 1DTF dives to zero intensity at 1 day, due to the cloud emission anisotropy.

It is important to understand that the differences between the data and the model 1DTFs at small lags could, at least in part, be due to resolution of the data 1DTF, i.e., the inversion process which recovers the data 1DTF is subject to the temporal resolution of the discrete data set and the signal-to-noise ratio of the light curves. In order to compare the model and data 1DTFs, we follow the prescription of Wanders et al. in which the model 1DTF is first degraded by convolving it with a Gaussian having a 3.8 day standard deviation (dashed line in Fig. 10). We find that, as before, the resulting 1DTF trails off toward zero near 28 days, but the amplitude at zero lag is at 0.52 . The peak and the centroid are shifted to about 7 and 9.2 days, which are near the shoulder of the data 1DTF in Wanders et al. In principle, the addition of a rapidly responding, more isotropic emission component to the model should introduce response at small lags and cause the present peak of the 1DTF to appear as a shoulder or as a secondary peak. We did not pursue this avenue, however.

Done \& Krolik (1996) also used the K95 campaign to derive the 1DTFs. Whether a shoulder is present in their 1DTFs depends on the choice of optimization parameters and whether they use only the HST or the combined $H S T / I U E$ data sets, i.e., a shoulder is present when only the HST data are used. In both cases the peak of the 1DTF occurs at zero lag. However, temporal structures of less than 2 days are not resolved, so it is uncertain if the peak is at zero days or at a slightly later time.

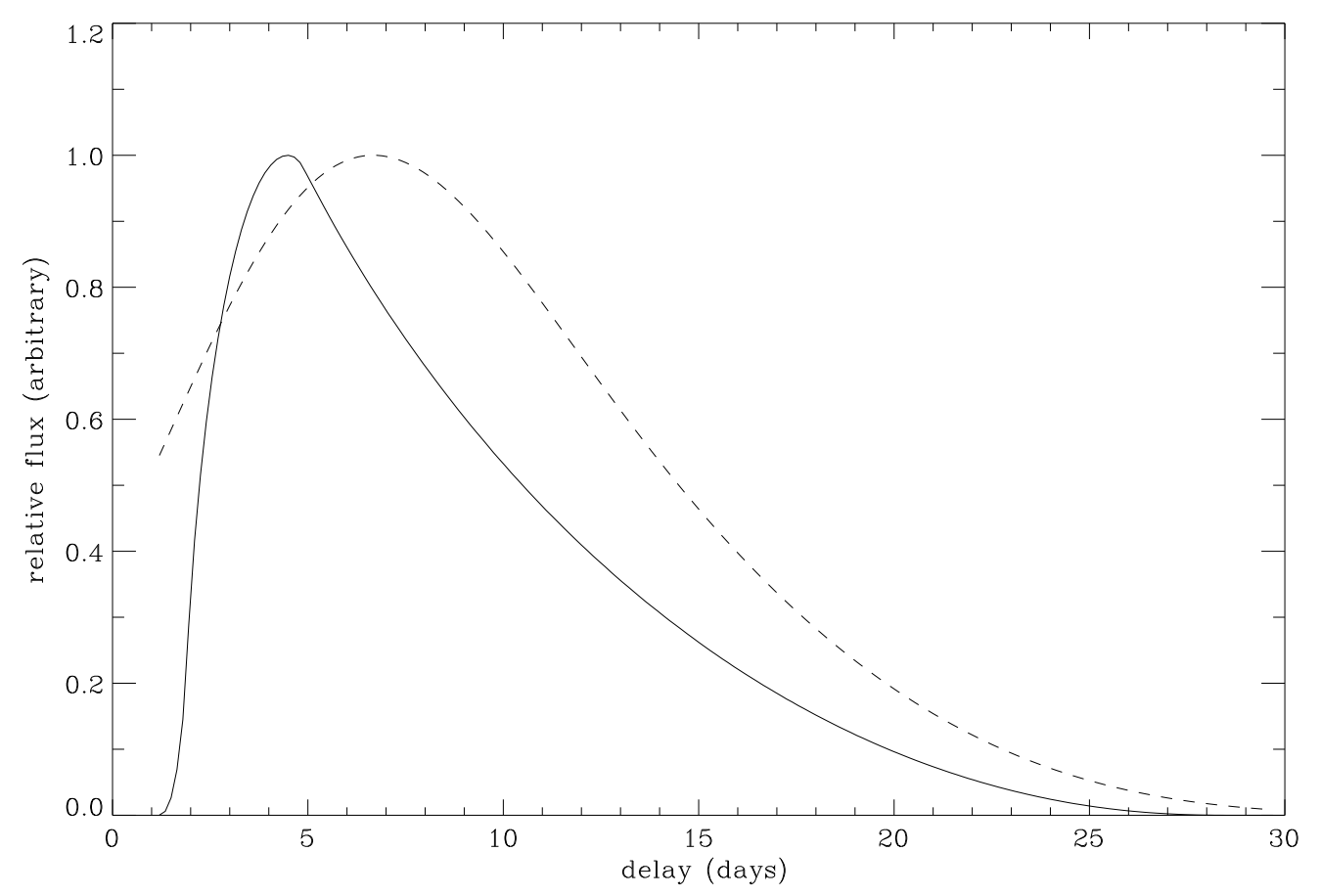

FIG. 10. - The 1DTF for the model, obtained by collapsing the 2DTF (Fig. 8a) along the velocity axis and normalized to the peak at 4 days. The continuous line represents the $1 \mathrm{DTF}$ using a 0.1 day pulse; the dashed line represents the 1DTF convolved with a 3.8 day Gaussian. 
Peterson (1993) presents response functions for a variety of emission lines, including C IV. The 1DTFs were extracted from the C91 campaign and are an updated version of the results published in Krolik et al. (1991). The 1DTF of C IV is peaked at zero lag and descends to a low plateau at 25 days. The amplitude drops off to zero at 100 days. There is a small secondary peak between 150 and 200 days, but it is thought to be an artifact due to correlations between the three separate events of the C91 campaign. Our model is consistent with the response function for $\mathrm{C}$ IV in Peterson (1993).

The above discussion focuses on the difference between our model and the data-derived response functions at small lags as being possibly due to the temporal resolution of the inversion methods. Residual differences between the model and the data 1DTFs at small lags can also be understood if low column density clouds are present in the flow. Such clouds might emit a fraction of their $\mathrm{C}$ IV line flux from the backsides, which is neglected in the present work. Response at zero (or small) lags, as indicated by the data 1DTF, means that there is emitting material along or close to the observer's line of sight.

\subsection{The Mean Line Profile}

Figure 11 presents the mean line profiles of the data and the model for the K95 campaign. The mean line profiles were generated by averaging the line profiles for the data and the model during the HST portion of the K95 campaign. The model mean line profile is also generated by integrating the $2 \mathrm{DTF}$ along the time axis. When the contribution of the NLR is removed from the model and compared to the time integration of the 2DTF, we find that they are identical, providing an independent check on the calculation of the 2DTF.

The essential features of the mean data profile are reproduced by the mean model profile. The differences are partly due to the absorption which is present in the blue part of the data line profile (i.e., in the $\mathrm{BC}$ ) and the contamination with $\mathrm{He}$ II and possible Fe II emissions in the red wing.

In the $\mathrm{RC}$ and between the $\mathrm{BC}$ and the $\mathrm{MBW}$, the model shows some excess of emission above the data. Additional emission can be explained in terms of our assumption that all the clouds are optically thick. Being efficient emitters, optically thick clouds produce more emission compared to the optically thin clouds, and our assumption is especially vulnerable at the inner and upper boundaries of the calculated BLR model. Realistically, optically thin clouds are likely to be found here, especially when the continuum is bright. Note, that the excess emission is not symmetrically placed with respect to the zero relative velocity shift. The red excess lies within the $\mathrm{RC}$ whereas the blue excess straddles the BC and the MBW. This argues against this excess emission originating close to the disk because the velocity field there is dominated by the Keplerian rotation. In this case, the excess emission would be symmetrically placed with respect to the zero relative frequency shift. Hence, the additional emission must come from the upper portion of the BLR, where the outflow dominates and where much of the low column density, optically thin (dustless) material is expected to be found. Exactly this can be seen in the dissections presented in Figure 9, where, at the distance of more than two light-days above the disk, the BLR contains only RC-, BC- and MBW-emitting material.

The model FBW does not fit the data well. Between the intrinsic and Galactic C IV absorption features the data mean line profile can be modeled with a single power-law fit, which breaks down between $-5400 \mathrm{~km} \mathrm{~s}^{-1}$ and -9600 $\mathrm{km} \mathrm{s}^{-1}$. This is in contrast with the red side which can be fitted with a single power law, all the way into the core, up to the NLR part. The above velocity interval is perhaps contaminated by $\mathrm{Si}$ II emission. However, the degree of contamination to the $\mathrm{C}$ IV emission is uncertain and so the modeling there represents a compromise between extreme cases.

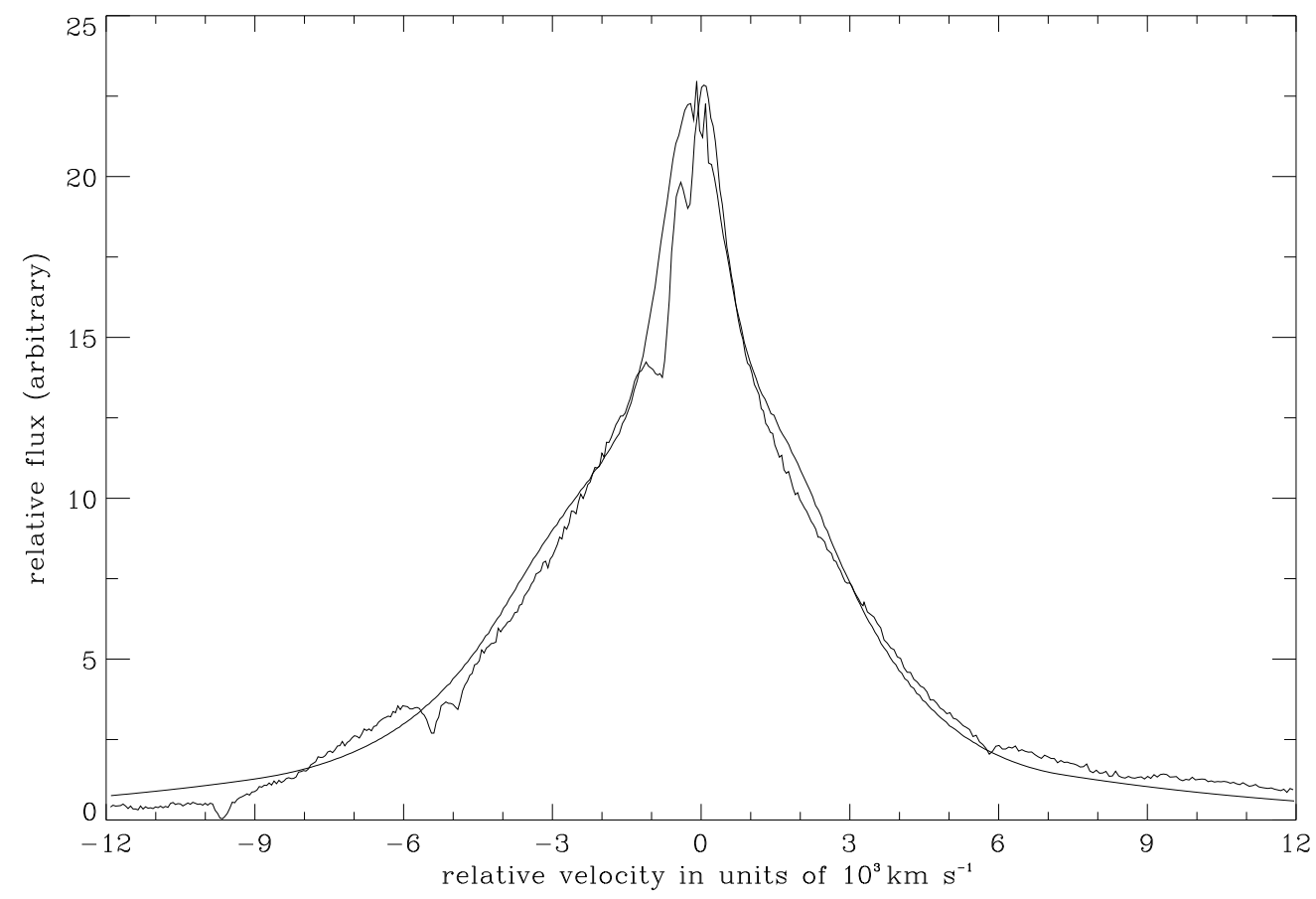

FIG. 11. - Comparison of the mean line profiles of the data and the model during the K95 campaign (includes the NLR contribution). The BLR contribution was obtained from the $2 \mathrm{DTF}$ (Fig. 8a) collapsed along the time axis. 
The model mean line profile also shows a slight $\sim 10 \%$ deficit of emission in the FRW. Part of the FRW is accounted for, because contamination corrections for He II and $\mathrm{Fe}$ II have not been subtracted off the mean data line profile (note that coarse corrections for these elements have been applied to the line segments mentioned earlier in $\S$ 4.2.1). In addition, the broad $\mathrm{He}$ II emission varies on a shorter timescale than the genuine C IV FRW (as clearly seen in the Fig. $6 d$ for the FRW prior to JD 2,449,115). Its modeling is outside the scope of this paper.

\subsection{Limitations of the Model}

In addition to the simplification of the emission anisotropy, there are other issues that need to be discussed. First, the EBS MHD model is a self-similar model providing scaling relationships for hydromagnetic flow and cloud parameters. We avoid a self-consistent calculation of $\mathrm{C}$ IV volume emissivity due to its complexity. Instead, we use a mix of cloud properties which is qualitatively consistent with the properties prescribed by the EBS model. The CLOUDY-calculated emissivity function reflects our assumption that the clouds are optically thick throughout the BLR. This necessarily ignores the thick-to-thin transition that some clouds will experience in response to the increase in the central continuum. Such a transition would ultimately lead to overionized clouds, to decreased cloud $\mathrm{C}$ IV emissivity and to a change in the cloud emission anisotropy (partly due to the dust destruction), modifying the response times of the BLR to continuum variations.

Second, the cloud emissivity function depends on the (unknown) ionizing continuum flux the clouds are subject to. Inspection of the continuum windows in K95 (see Fig. 6 of K95) reveals that at shorter wavelengths the continuum variations become larger and the peaks and valleys of the light curves become sharper. If this trend continues to even shorter wavelengths, the relevant ionizing continuum variations could be significantly different in character from what is being used here.

Third, the overall geometry of the $\mathrm{C}$ IV emitting region was assumed to be time-independent. This certainly is an approximation, as we expect that the upper and outer boundaries of the emitting region to move in response to the continuum variations. In particular, the opening angle of the wind which is fixed now at $120^{\circ}$ should vary because clouds close to the upper boundary may become overionized and perhaps destroyed. The outer boundary of the C IV emitting region, set by the condition $\tau_{\mathrm{obs}}=1$, will move as well because the obscuration will change with the variations in the ionizing continuum.

A related issue arises from the approximation that has been made in the radiation transfer through the cloud system. As mentioned in $\S 3$, clouds in the model have sufficient optical depth to obscure the central continuum source in some directions. The purely geometrical obscuration used assures that the clouds either see all or a fraction of the original continuum whose shape was unaltered. A more realistic model should account for the continuum reprocessing by the clouds and in particular the changing continuum shape. The latter will affect the cloud spectrum and response to the continuum input. Accounting for this effect, however, requires the full solution of the radiative transfer problem which is outside the scope of this paper.

Finally, the model presented in this paper was not fully optimized, so, in principle, better fits to line profiles and measured time lags are possible. However, we have shown that the hydromagnetic wind model is capable of reproducing the main features observed in both campaigns. A more detailed analysis is the subject of a future work.

\section{CONCLUSIONS}

We attempt to infer the dynamical state of the BLR in the Seyfert 1 galaxy NGC 5548 by analyzing results of the longterm observations and by providing a critical comparison with the predictions of a hydromagnetically driven outflow model from an optically thick accretion disk. Our modeling is based on the EBS model, subject to a number of modifications, i.e., cloud emission anisotropy and cloud-cloud obscuration. We have shown that a model of a rotating magnetized wind from a disk is compatible with the observed line profiles, and the BLR line and continuum variability monitored in C91 and K95 campaigns.

We have demonstrated that a time series of synthetic $\mathrm{C}$ IV emission lines can be fitted to the HST-observed line profiles reasonably well, using the observed UV continuum light curve as the driver and without adjusting the model parameters. Our best model for the observed $\mathrm{C}$ IV emission in NGC 5548 consists of a disklike region extending $\pm 30^{\circ}$ in the polar directions from the equatorial plane and between 1 1t-day and 24 1t-day in the radial direction. Our model is also highly anisotropic because radiation is essentially backscattered. The C IV-emitting part of the wind is bounded by the molecular wind at larger radii, depending on the altitude above the disk. We expect the low-ionization lines, like $\mathrm{Mg}$ II, to come from the region where the central UV soft-X-ray continuum is attenuated, i.e., from $\tau_{\text {obs }}>1$. The best fit to the observed $\mathrm{C}$ IV line profiles also constrained the mass of the central black hole to $\sim 3 \times 10^{7}$ $M_{\odot}$. Surprisingly, the variable integrated line flux calculated within the model also fits reasonably well that from the IUE (C91) campaign which happened $4 \mathrm{yr}$ prior to the HST campaign, again without any parameter adjustments or flux renormalization.

We have divided the synthetic $\mathrm{C}$ IV line profile into six segments corresponding to the blue and red cores, and mid and far-wings, and calculated the cross-correlations between different line segments as well as between the line segments and the continuum. The model predicts that the mid-red wing response precedes the responses of all other $\mathrm{C}$ IV line segments, and that the wings respond before the line cores. Given that no adequate method in computing the errors for data lags and centroids exists in the literature, the data cross-correlation function provides results which appear inconclusive, making any direct comparison with the model premature. The only statement we make in comparing the data lags and centroids with the model is that they are compatible.

In order to understand the model response, the underlying geometry and the $\mathrm{C}$ IV emission distribution in the velocity field in the model BLR, we have computed the one-dimensional and two-dimensional transfer functions. We find that, due to the cloud emission anisotropy, an observer at a typical aspect angle of $40^{\circ}$ to the rotation axis receives the main contribution to the $\mathrm{C}$ IV line coming from the backside of the wind. Consequently, at the base of the wind, the emitting gas kinematics is dominated by a rotation plus an outflow velocity projected away from the observer. It is the shape of the streamlines in the $r z$-plane (parabolas), which leads to a switch from the redshifted 
outflow velocities to the blueshifted ones at some height above the disk. Initially, the red part of the $\mathrm{C}$ IV line grows faster than the blue one. The steepest decline in the redshifted emission appears in the mid-red wing which turns over first. This predicts a differential response across the $\mathrm{C}$ IV profile, in particular, a redward asymmetric response. Unfortunately, no unambiguous differential lags have been measured across the $\mathrm{C}$ IV line during the HST part of the K95 campaign, and so the resolution of this problem awaits further observations.

The idea that the outer parts of accretion disks in AGNs can be molecular is supported by detection of such gas in the inner regions of AGNs (Scoville et al. 1989) and by the possibility that all AGNs are fueled by a dusty and molecular galactic ISM (Shlosman, Frank, \& Begelman 1989). If this material can be raised above the disk, it can easily obscure the central source by intercepting and reprocessing a large fraction of the UV-soft-X-ray luminosity into the infrared. Seyfert 2 galaxies, with NGC 1068 as their prototype, fall within this category of objects having intervening column densities in excess of $10^{25} \mathrm{~cm}^{-2}$ (Krolik \& Begelman 1988; Mulchaey, Mushotzky, \& Weaver 1992). Hard $\mathrm{X}$-rays will be least affected and hence may serve as diagnostic of AGNs viewed through a large column of obscuring material almost supressing the BLRs, e.g., in the so-called narrow-line X-ray galaxies (e.g., Lawrence 1991). The possibility that the obscuration comes from the highcolumn density material in the hydromagnetic wind was used by EBS to explain LILs and the broad absorption-line quasars, and was incorporated by Königl \& Kartje (1994) into the unification scheme of the AGNs. Adopting the wind origin of the obscuration resolves the problem of vertical support for molecular tori in Seyfert galaxies, described by Krolik \& Begelman (1988).

In this work we have largely neglected the dynamical effects of radiation pressure from the central continuum on the magnetized wind. Although this effect cannot be ignored in brightest AGNs, as shown by de Kool \& Begelman (1995) for the case of broad absorption-line quasars, it might not dominate the cloud dynamics in lower luminosity Seyfert galaxies. This is demonstrated by an apparent survival of the molecular torus in NGC 1068 which is geometrically thick and has an opening angle which is only slightly smaller than the angle of $120^{\circ}$ inferred here for NGC 5548. Along with Königl \& Kartje, we do expect that central radiation flux will control the opening angle of the wind, which is consistent with observation that the thickness of molecular tori in Seyfert galaxies seem to decrease with increasing central luminosities (Lawrence 1991; Miller 1994). It is unclear whether the wind opening angle can respond to short-time variability of the central continuum, such as observed in NGC 4151 which changed its appearance from Seyfert 1 to Seyfert 1.9, as the continuum luminosity decreased (Clavel et al. 1990; Ulrich et al. 1991).

In summary, this model provides an attempt to match a dynamical model to the monitoring campaigns of NGC 5548 . We have avoided a systematic parameter search over the entire phase space available for modeling, so the model presented here is neither guaranteed to be unique or to provide an optimal fit to the data. However, we conclude, based on our analysis, that a rotating outflow from an accretion disk provides a viable model for the BLR in NGC 5548 , in particular, and for other AGNs, in general.

We thank Mitch Begelman, Chris Done, Matthias Dietrich, Julian Krolik, and Brad Peterson for valuable discussions, and the anonymous referee for helpful comments. We are grateful to Gary Ferland for assistance with the recent version of CLOUDY. This work was supported in part by NASA grants WKU-521751-94-08 and 521752-9506 and NAGW-3839 (I. S.), NAG 3-223 (K. T. K.), and NSF grants AST 92-23370 and AST 95-29170 (R. D. B.).

\section{REFERENCES}

Baldwin, J. A., Ferland, G. J., Korista, K. T., \& Verner, D. 1995, ApJ, 455, L119

Blandford, R. D., \& McKee, C. F. 1982, ApJ, 255, 419

Blandford, R. D., \& Payne, D. G. 1982, MNRAS, 199, 883

Cassidy, I., \& Raine, D. J. 1996, MNRAS, submitted

Chiang, J., \& Murray, N. 1996, ApJ, submitted

Clavel, J., et al. 1990, MNRAS, 246, 668

Clavel, J., et al. 1991, ApJ, 366, 64 (C91)

Collin-Souffrin, S., \& Lasota, J.-P. 1989, PASP, 100, 1041

Corbin, M. R. 1990, ApJ, 357, 346

Crenshaw, D. M., \& Blackwell, J. H. 1990, ApJ, 358, L37

Crenshaw, D. M., Boggess, A., \& Wu, C.-C. 1993, ApJ, 416, L67

de Kool, M., \& Begelman, M. C. 1995, ApJ, 455, 448

Dietrich, M., et al. 1993, ApJ, 408, 416

Done, C., \& Krolik, J. H. 1996, ApJ, 463, 144

Emmering, R. T., Blandford, R. D., \& Shlosman, I. 1992, ApJ, 385, 460 (EBS)

Espey, B. R., Carswell, R. F., Bailey, J. A., Smith, M. G., \& Ward, M. J. 1989, ApJ, 342, 666

Fabian, A. C., Guilbert, P., Arnaud, K., Shafer, R., Tenant, A., \& Ward, M. 1986, MNRAS, 280, 574

Ferland, G. J. 1994, Hazy: A Brief Introduction to CLOUDY (Lexington: Univ. Kentucky, Dept. Physics \& Astronomy Int. Rep.

Ferland, G. J., Korista, K. T., \& Peterson, B. M. 1990, ApJ, 363, L21

Ferland, G. J., Peterson, B. M., Horne, K., Welsh, W. F., \& Nahar, S. N. 1992, ApJ, 387, 95

Gaskell, C. M. 1982, ApJ, 263, 79

Gaskell, S. M., \& Peterson, B. M. 1987, ApJS, 65, 1

Gondhalekar, P. M., Horne, K., \& Peterson, B. M. eds. 1994, Reverberation Mapping of the Broad-Line Region in AGNs (ASP Conf. Proc. 69)

Horne, K., Welsh, W. F., \& Peterson, B. M. 1991, ApJ, 367, L5

Königl, A., \& Kartje, J. F. 1994, ApJ, 434, 446

Koratkar, A. P., \& Gaskell, C. M. 1991, ApJ, 345, 637

Korista, K. T., et al. 1995, ApJS, 97, 285 (K95)

Krolik, K. T., \& Begelman, M. C. 1988, ApJ, 329, 702
Krolik, K. T., \& London, R. A. 1983, ApJ, 267, 18

Krolik, J. H., Horne, K., Kallman, T. R., Malkan, M. A., \& Edelson, R. A. 1991, ApJ, 371, 541

Kwan, J., \& Carrol, T. J. 1982, ApJ, 261, 25

Lawrence, A. 1991, MNRAS, 252, 586

Maoz, D., et al. 1990, ApJ, 351, 75

Maoz, D., et al. 1993, ApJ, 404, 576

Mathews, W. G. 1982, ApJ, 258, 425

.1986, ApJ, 305, 187

Mathews, W. G., \& Capriotti, E. R. 1985, in Proc. Santa Cruz Workshop on Astrophysics of Active Galaxies and QSOs, ed. J. S. Miller (Mill Valley, CA: Science Books), 185

Mathews, W. G.. \& Ferland, G. J. 1987, ApJ, 258, 425

Miller, J. S. 1994, in Proc. I Stromlo Symp. on Physics of Active Galaxies, ed. V. Bicknell et al. (ASP Conf. Proc. 54), 149

Mulchaey, J. S., Mushotzky, R. F., \& Weaver, K. A. 1992, ApJ, 390, L69

Murray, N., Chiang, J., Grossman, S., \& Voit, G. M. 1996, ApJ, in press

Netzer, H. 1990, in Active Galactic Nuclei, ed. T. J.-L. Courvoisier \& M. Mayor (Berlin: Springer)

Osterbrock, D. E. 1996, in The Violent Universe: Key Problems in Astronomy \& Astrophysics, ed. G. Münch et al. (Cambridge: Cambridge Univ. Press), in press

Pérez, E., Robinson, A., \& de la Fuente, L. 1992a, MNRAS, 255, 502

.1992b, MNRAS, 256, 110

Peterson, B. M. 1987, ApJ, 312, 79

. 1993, PASP, 105, 247

Peterson, B. M., Korista, K. T., \& Cota, S. A. 1987, ApJ, 312, L1

Peterson, B. M., Reichert, G. A., Korista, K. T., \& Wagner, R. M. 1990, ApJ, 352, 68

Peterson, B. M., et al. 1991, ApJ, 368, 119

. 1993, ApJ, 402, 469

Phinney, E. S. 1989, in Theory of Accretion Disks, ed. F. Meyer et al. (Dordrecht: Kluwer), 457

Rees, M. J. 1987, MNRAS, 228, 47P

Robinson, A. 1995, MNRAS, 272, 647 
Rosenblatt, E., \& Malkan, M. A. 1990, ApJ, 350, 132

Sanders, D. B., Phinney, E. S., Neugebauer, G., Soifer, B. T., \& Matthews, K. 1989, ApJ, 347, 29

Scoville, N. J., Sanders, D. B., Sargent, A. I., Soifer, B. T., \& Tinney, C. G. 1989, ApJ, 345, L25

Shields, G. A. 1978, in Pittsburgh Conf. on BL Lac Objects, ed. A. M. Wolfe (Pittsburgh: Univ. of Pittsburgh Press), 257

Shlosman, I., \& Begelman, M. C. 1989, ApJ, 341, 685

Shlosman, I., Frank, J., \& Begelman, M. C. 1989, Nature, 338, 45

Shlosman, I., Vitello, P., \& Shaviv, G. 1985, ApJ, 294, 96

Stirpe, G. M., de Bruyn, A. G., \& van Groningen, E. 1988, A\&A, 200, 9
Ulrich, M.-H., et al. 1991, ApJ, 382, 483

Walter, R., et al. 1994, A\&A, 285, 119

Wamsteker, W., et al. 1990, ApJ, 354, 446

Wanders, W., et al. 1995, ApJ, 453, L87

Wanders, W., \& Peterson, B. M. 1996, ApJ, 466, 174

Welsh, W. F., \& Horne, K. 1991, ApJ, 379, 586

Weymann, R. J., Scott, J. S., Schiano, A. V. R., \& Christiansen, W. A. 1982, ApJ, 262, 497

White, R. J., \& Peterson, B. M. 1994, PASP, 106, 879

Wilkes, B. J. 1984, MNRAS, 207, 73 